\title{
Spaces of Bessel-potential type and embeddings: the super-limiting case
}

\author{
Júlio S. Neves*1 \\ ${ }^{1}$ Departamento de Matemática da FCT, Universidade de Coimbra, Apartado 3008, 3001-454 Coimbra, Portugal
}

Received 18 April 2002, accepted 24 June 2002

Published online 12 January 2004

\begin{abstract}
Key words Lorentz-Karamata spaces, Bessel-Lorentz-Karamata spaces, Besov-Lipschitz-Karamata spaces MSC (2000) 26A12, 46E30, 46E35, 26A15, 26A16, 47B38, 26D 10, 26D 15

We consider Bessel-potential spaces modelled upon Lorentz-Karamata spaces and establish embedding theorems in the super-limiting case. In addition, we refine a result due to Triebel, in the context of Bessel-potential spaces, itself an improvement of the Brézis-Wainger result (super-limiting case) about the "almost Lipschitz continuity" of elements of $H_{p}^{1+n / p}\left(\mathbb{R}^{n}\right)$. These results improve and extend results due to Edmunds, Gurka and Opic in the context of logarithmic Bessel potential spaces. We also give examples of embeddings of Besselpotential type spaces which are not of logarithmic type.
\end{abstract}

(c) 2004 WILEY-VCH Verlag GmbH \& Co. KGaA, Weinheim

\section{Introduction}

This paper is a continuation of [19], where we established (limiting) embeddings of Bessel-potential spaces modelled upon appropriate Lorentz-Karamata (LK) spaces $X=L_{p, q ; b}\left(\mathbb{R}^{n}\right)$ into either LK spaces or Orlicz spaces.

Here we deal with the super-limiting case. For Sobolev spaces $W_{p}^{k}(\Omega)$, the Sobolev classical embedding theorem asserts that if $p>n / k$ and $\Omega \subset \mathbb{R}^{n}$ is a domain with a sufficiently smooth boundary, then $W_{p}^{k}(\Omega) \hookrightarrow$ $C(\Omega)$, where $C(\Omega)$ is the space of scalar-valued bounded continuous functions on $\Omega$. However, in this case more can be said, as embeddings in Hölder spaces $C^{0, \alpha}(\bar{\Omega})$ are possible. For example, $W_{p}^{k}(\Omega) \hookrightarrow C^{0, \alpha}(\bar{\Omega})$, for all $\alpha \in(0,1)$, when $k=1+n / p \in \mathbb{N}$ and $\Omega \subset \mathbb{R}^{n}$ is a domain with a sufficient smooth boundary, cf. e.g. [1, Lemmas 5.17]. In the particular case $k=1+n$ and $p=1$, the previous embedding can be improved as the Sobolev space is embedded into the Lipschitz space. Therefore, we may ask if an embedding into a Lipschitz space for $p>1$ and $k=1+n / p \in \mathbb{N}$ would be possible. The answer is negative; see [8, Theorem 3.3] where a more general result is established, which deals with the sharpness of embeddings of logarithmic Bessel potential spaces into general Hölder spaces. This result for Sobolev spaces is common knowledge, but as remarked in [8] it is hard to find a precise argument in the literature: [1, Example 5.28] does not settle the question as there is a slip in the calculations, cf. [8, Remark 5.2]. However, Brézis-Wainger (cf. [4, Corollary 5]) proved that elements of the Bessel potential space $H_{p}^{1+n / p}\left(\mathbb{R}^{n}\right)$, with $1<p<+\infty$, are "almost Lipschitz continuous", i.e., $H_{p}^{1+n / p}\left(\mathbb{R}^{n}\right) \hookrightarrow \operatorname{Lip}_{\infty, \infty}^{\left(1,-\frac{1}{p^{\prime}}\right)}\left(\mathbb{R}^{n}\right)$, which implies, for some positive constant $c$,

$$
|f(x)-f(y)| \leq c\left\|f\left|H_{p}^{1+n / p} \|\right| x-\left.y|| \log |x-y|\right|^{\frac{1}{p^{\prime}}},\right.
$$

for all $f \in H_{p}^{1+n / p}\left(\mathbb{R}^{n}\right)$ and $x, y \in \mathbb{R}^{n}$ such that $0<|x-y|<\frac{1}{2}$.

When the more general space $H_{p}^{1+n / p} X$, with $1<p<+\infty$, where for example $X=L^{p}(\log L)^{a}\left(\mathbb{R}^{n}\right)$ is a Zygmund space $(a \in \mathbb{R})$, is considered, Edmunds, Gurka and Opic [7] proved the following: if $a>\frac{1}{p^{\prime}}$, the fractional Sobolev-type space $H^{1+n / p} X$ is embedded into the Lipschitz space $\operatorname{Lip}\left(\mathbb{R}^{n}\right)$; if $a<\frac{1}{p^{\prime}}$, the space $H^{1+n / p} X$ is embedded into the Lipschitz-type space (general Hölder-type space) $\operatorname{Lip}_{\infty, \infty}^{\left(1, a-\frac{1}{p^{\prime}}\right)}\left(\mathbb{R}^{n}\right)$, which

\footnotetext{
* e-mail: jsn@mat.uc.pt
} 
entails, for some positive constant $c$,

$$
|f(x)-f(y)| \leq c\left\|f\left|H_{p}^{1+n / p} X \|\right| x-y|| \log |x-y|^{\frac{1}{p^{\prime}}-a},\right.
$$

for all $f \in H_{p}^{1+n / p} X$ and $x, y \in \mathbb{R}^{n}$ such that $0<|x-y|<\frac{1}{2}$. The same authors also dealt with the case $a=\frac{1}{p^{\prime}}$, where the target space for the embedding is a double logarithmic Lipschitz-type space. We refer to [7] for more general embedding results of the Bessel-potential spaces modelled upon generalised Lorentz-Zygmund spaces into generalised Hölder spaces. Nevertheless, these results can be improved for $a \leq \frac{1}{p^{\prime}}$. A smaller Lipschitz space, in the sense of inclusions, as a target space can be found. When $a=0$, i.e., $H^{1+n / p} X$ is the fractional Sobolev space $H_{p}^{1+n / p}\left(\mathbb{R}^{n}\right)$, Triebel [22, Theorem 14.2 (ii)] showed that $H_{p}^{1+n / p}\left(\mathbb{R}^{n}\right)$ is embedded into the space of Lipschitz type $\operatorname{Lip}_{\infty, p}^{(1,-1)}\left(\mathbb{R}^{n}\right)$, which is smaller in the sense of inclusions than the space $\operatorname{Lip}_{\infty, \infty}^{\left(1,-\frac{1}{p^{\prime}}\right)}\left(\mathbb{R}^{n}\right)$, improving in this way the above result of Brézis-Wainger (super-limiting case). Note that Triebel's result is established for the Triebel-Lizorkin spaces $F_{p, q}^{1+n / p}\left(\mathbb{R}^{n}\right)$, with $1<p<+\infty$ and $0<q \leq+\infty$, which coincides with the Bessel-potential space $H_{p}^{1+n / p}\left(\mathbb{R}^{n}\right)$ when $q=2$. Motivated by this result of Triebel, we prove that, if $a<\frac{1}{p^{\prime}}$, the fractional Sobolev-type space $H^{1+n / p} X$ is embedded into the Lipschitz-type space $\operatorname{Lip}_{\infty, p}^{(1, a-1)}\left(\mathbb{R}^{n}\right)$, which is smaller in the sense of inclusions than the space $\operatorname{Lip}_{\infty, \infty}^{\left(1, a-\frac{1}{p^{\prime}}\right)}\left(\mathbb{R}^{n}\right)$, improving in this way the result mentioned due to Edmunds, Gurka and Opic; the case $a=\frac{1}{p^{\prime}}$ is also covered by the appearance of a double logarithmic Lipschitz-type space as a target space smaller than that considered in [7]; see Corollary 5.18 and Remark 5.19(ii). This is a consequence of our more general result given by Theorem 5.16, which concerns embeddings of Bessel-potential spaces modelled upon Lorentz-Karamata into spaces of Lipschitz type.

In Section 2 we present some notation.

Section 3 deals with some properties of slowly varying functions and results about Lorentz-Karamata spaces.

In Section 4, the Besov-Lipschitz-Karamata spaces $\Lambda_{p, q}^{\lambda, b}\left(\mathbb{R}^{n}\right), 1 \leq p \leq+\infty, 0<q \leq+\infty, 0<\lambda \leq 1$ and $b$ a slowly varying function, are considered.

In the last section we consider Bessel-potential spaces modelled upon LK spaces, and referred in what follows as Bessel-Lorentz-Karamata (BLK) spaces, and we establish embeddings theorems in the super-limiting case, where the main result is given by Theorem 5.16. We also present examples of embeddings of BLK spaces which are not of logarithmic type. These examples have not been considered before in the literature, as far as we are aware.

\section{Notation and preliminaries}

As usual, $\mathbb{R}^{n}$ denotes Euclidean $n$-dimensional space. Let $(R, \Sigma, \mu)$, usually denoted by $(R, \mu)$, be a totally $\sigma$-finite measure space, referred to in the sequel only as a measure space. When $R=\mathbb{R}^{n}$ we shall always take $\mu$ to be the Lebesgue measure $\mu_{n}$. The family of all extended scalar-valued (real or complex) $\mu$-measurable functions on $R$ will be denoted by $\mathcal{M}(R, \mu) ; \mathcal{M}_{0}(R, \mu)$ will stand for the subset of $\mathcal{M}(R, \mu)$ consisting of all those functions which are finite $\mu$-a.e.; and $\mathcal{M}^{+}(R, \mu)\left(\mathcal{M}_{0}^{+}(R, \mu)\right)$ will stand for the subset of $\mathcal{M}(R, \mu)$ $\left(\mathcal{M}_{0}(R, \mu)\right)$ consisting of all those functions which are non-negative $\mu$-a.e.

Let $f \in \mathcal{M}_{0}(R, \mu)$. The non-increasing rearrangement of $f$ is the function $f^{*}$ defined on $[0,+\infty)$ by $f^{*}(t)=\inf \{\lambda \geq 0: \mu\{x \in R:|f(x)|>\lambda\} \leq t\}$ for all $t \geq 0$, and the maximal function $f^{* *}$ of $f^{*}$ is defined by $f^{* *}(t)=(1 / t) \int_{0}^{t} f^{*}(s) d s$ for all $t>0$.

For general facts about (rearrangement-invariant) Banach function spaces with Banach function norm (or simply a function norm) $\rho$ over a measure space $(R, \mu)$ we refer to [2, Chap. 1, Chap. 2]. Nevertheless, let us recall the concept of absolutely continuous norm, for the convenience of the reader. If $X$ is a Banach function space over $(R, \mu)$, a function $f$ of $X$ is said to have absolutely continuous norm in $X$ if $\left\|f \chi_{E_{n}}\right\|_{X} \rightarrow 0$ for every sequence $\left\{E_{n}\right\}_{n \in \mathbb{N}}$ of subsets of $R$ such that $E_{n} \rightarrow \emptyset \mu$-a.e. (that is, $\chi_{E_{n}} \rightarrow 0 \mu$-a.e.); if every element $f$ of $X$ has absolutely continuous norm, $X$ is said to have absolutely continuous norm. Proposition I.3.2 in [2], shows us that regarding the absolutely continuity of a function in a Banach function space it is enough to restrict our attention to decreasing sequences $\left\{E_{n}\right\}_{n \in \mathbb{N}}$.

Let $p \in(0,+\infty]$. We denote by $L_{p}(R)$ the Lebesgue space endowed with the (quasi-) norm $\|\cdot\|_{p ; R}$. 
The space of all scalar-valued (real or complex), bounded and continuous functions on $\mathbb{R}^{n}$ is denoted by $C\left(\mathbb{R}^{n}\right)$ and it is equipped with the $L_{\infty}\left(\mathbb{R}^{n}\right)$ norm. By $C\left(\overline{\mathbb{R}^{n}}\right)$ we mean the subspace of $C\left(\mathbb{R}^{n}\right)$ of the uniformly continuous functions, whereas $C^{1}\left(\overline{\mathbb{R}^{n}}\right)$ denotes the space of all the functions $f \in C\left(\overline{\mathbb{R}^{n}}\right)$ such that the $n$ partial derivatives of order $1, \frac{\partial f}{\partial x_{j}}(j=1, \ldots, n)$, are also in $C\left(\overline{\mathbb{R}^{n}}\right)$ and it is endowed with the norm

$$
\|f\|_{C^{1}}:=\|f\|_{\infty}+\sum_{j=1}^{n}\left\|\frac{\partial f}{\partial x_{j}}\right\|_{\infty} .
$$

Let $\mathcal{S}\left(\mathbb{R}^{n}\right)$ be the Schwartz space of all scalar-valued rapidly decreasing infinitely differentiable functions $u$ on $\mathbb{R}^{n}$ (usually we write $\mathcal{S}$ instead of $\mathcal{S}\left(\mathbb{R}^{n}\right)$ ).

For each $h \in \mathbb{R}^{n}$, the difference operator $\Delta_{h}$ is defined on scalar functions $f$ on $\mathbb{R}^{n}$ by $\left(\Delta_{h} f\right)(x)=$ $f(x+h)-f(x)$ for all $x \in \mathbb{R}^{n}$.

In what follows we use the notation $Y_{p}\left(\mathbb{R}^{n}\right)=L_{p}\left(\mathbb{R}^{n}\right)$, if $1 \leq p<+\infty$, and $Y_{\infty}\left(\mathbb{R}^{n}\right)=C\left(\mathbb{R}^{n}\right)$. Let $p \in[1,+\infty]$. The modulus of smoothness of a function $f$ in $Y_{p}\left(\mathbb{R}^{n}\right)$ is defined by

$$
\omega(f, t)_{p}=\sup _{|h| \leq t}\left\|\Delta_{h} f\right\|_{p} \text { for all } t \geq 0 .
$$

If $f \in C\left(\mathbb{R}^{n}\right)$, then $\omega(f, t)_{\infty} \rightarrow \omega(f, 0)_{\infty}=0$ as $t \downarrow 0$ if, and only if, $f \in C\left(\overline{\mathbb{R}^{n}}\right)$. Let $p \in[1,+\infty]$ and $f$ in $Y_{p}\left(\mathbb{R}^{n}\right)$. Let

$$
\widetilde{\omega}(f, t)_{p}=\frac{\omega(f, t)_{p}}{t} \text { for each } t>0
$$

then $\widetilde{\omega}(f, .)_{p}$ is equivalent to a non-increasing function on $(0,+\infty)$. We refer to [2, pp. 331-333] and to [5, pp. 40-50] for more details.

Now let $m \in \mathbb{N}$ and $\boldsymbol{\alpha}=\left(\alpha_{1}, \ldots, \alpha_{m}\right) \in \mathbb{R}^{m}$. We denote by $\boldsymbol{\ell}^{\boldsymbol{\alpha}}$ the real function defined by $\boldsymbol{\ell}^{\boldsymbol{\alpha}}(t)=$ $\prod_{i=1}^{m} \ell_{i}^{\alpha_{i}}(t)$ for all $t \in(0,+\infty)$, where $\ell_{1}, \ldots, \ell_{m}$ are positive functions defined on $(0,+\infty)$ by $\ell_{1}(t)=1+$ $|\log t|$, and, if $m \geq 2, \ell_{i}(t)=1+\log \ell_{i-1}(t), i \in\{2, \ldots, m\}$. For formal reasons, we put, if $m=0$, $\prod_{i=1}^{m} \ell_{i}^{\alpha_{i}}=\ell^{\boldsymbol{\alpha}}=1$.

The Bessel kernel $g_{\sigma}, \sigma>0$, is defined as that function on $\mathbb{R}^{n}$ whose Fourier transform is

$$
\widehat{g_{\sigma}}(\xi)=(2 \pi)^{-n / 2}\left(1+|\xi|^{2}\right)^{-\sigma / 2}, \quad \xi \in \mathbb{R}^{n},
$$

where the Fourier transform $\hat{f}$ of a function $f$ is given by $\hat{f}(\xi)=(2 \pi)^{-n / 2} \int_{\mathbb{R}^{n}} e^{-i \xi \cdot x} f(x) d x$. It is known that $g_{\sigma}$ is a positive, integrable function which is analytic except at the origin.

Let $m \in \mathbb{N}$. Given $\boldsymbol{\alpha}=\left(\alpha_{1}, \ldots, \alpha_{m}\right), \boldsymbol{\beta}=\left(\beta_{1}, \ldots, \beta_{m}\right) \in \mathbb{R}^{m}$ and $\sigma \in \mathbb{R}$, we shall use the convention $\boldsymbol{\alpha}+\boldsymbol{\beta}=\left(\alpha_{1}+\beta_{1}, \ldots, \alpha_{m}+\beta_{m}\right), \boldsymbol{\alpha}+\sigma=\left(\alpha_{1}+\sigma, \ldots, \alpha_{m}+\sigma\right), \sigma \boldsymbol{\alpha}=\left(\sigma \alpha_{1}, \ldots, \sigma \alpha_{m}\right)$. If $\boldsymbol{\alpha}=(0, \ldots, 0) \in \mathbb{R}^{m}$ we denote $\boldsymbol{\alpha}$ by $\mathbf{0}$. We write $\boldsymbol{\beta} \prec \boldsymbol{\alpha}$, or $\boldsymbol{\alpha} \succ \boldsymbol{\beta}$, if either $\beta_{1}-\alpha_{1}<0$ or there exists $k \in\{2, \ldots, m\}$ such that $\beta_{k}-\alpha_{k}<0$ and $\beta_{j}=\alpha_{j}, j=1, \ldots, k-1$. We use the symbol $\boldsymbol{\beta} \preceq \boldsymbol{\alpha}$, or $\boldsymbol{\alpha} \succeq \boldsymbol{\beta}$, to mean that either $\boldsymbol{\beta} \prec \boldsymbol{\alpha}$ or $\boldsymbol{\beta}=\boldsymbol{\alpha}$.

Let $p \in[1,+\infty], k \in\{1, \ldots, m\}$. We denote by $\boldsymbol{\delta}_{p ; m, k}$ the $m$-tuple $\left(\delta_{1}, \ldots, \delta_{m}\right)$, where $\delta_{i}=\frac{1}{p}, i=1, \ldots, k$, and $\delta_{i}=0$ for $i=k+1, \ldots, m$, if $k+1 \leq m$.

In the sequel, we let $c$ denote a positive constant. In a chain of inequalities $c$ may stand for several different constants if it is not important to distinguish between them, otherwise we use $c$ with subscripts. For two nonnegative expressions (i.e. functions or functionals) $\mathcal{A}, \mathcal{B}$, the symbol $\mathcal{A} \precsim \mathcal{B}$ means that $\mathcal{A} \leq c \mathcal{B}$, for some positive constant $c$ independent of the variables in the expressions $\mathcal{A}$ and $\mathcal{B}$. If $\mathcal{A} \precsim \mathcal{B}$ and $\mathcal{B} \precsim \mathcal{A}$, we write $\mathcal{A} \approx \mathcal{B}$. We adopt the convention that $a /+\infty=0$ and $a / 0=+\infty$ for all $a>0$. If $p \in[1,+\infty]$, the conjugate number $p^{\prime}$ is given by $1 / p+1 / p^{\prime}=1$.

\section{Slowly varying functions and Lorentz-Karamata spaces}

A positive and Lebesgue-measurable function $b$ is said to be slowly varying (s.v.) on $[1,+\infty)$ in the sense of Karamata, if for each $\epsilon>0, t^{\epsilon} b(t)$ is equivalent to a non-decreasing function on $[1,+\infty)$ and $t^{-\epsilon} b(t)$ is 
equivalent to a non-increasing function on $[1,+\infty)$; see Chapter I in [3] for a detailed study of the Karamata theory.

Properties and examples of s.v. functions can be seen in [23, Chapter V, pp. 186], [3], [9] and [19]. The following functions are s.v. on $[1,+\infty)$.

(i) $b(t)=\ell^{\boldsymbol{\alpha}}(t)$, with $\boldsymbol{\alpha} \in \mathbb{R}^{m}$.

(ii) $b(t)=\exp \left(\log ^{\alpha} t\right)$, with $0<\alpha<1$.

(iii) $b_{m}(t)=\exp \left(\ell_{m}^{\alpha}(t)\right)$, with $0<\alpha<1$ and $m \in \mathbb{N}$.

Note that if $m \geq 2$ in the last example, we may consider $\alpha=1$. In this case $b_{m} \approx \ell_{m-1}$.

Given a slowly varying function $b$ on $[1,+\infty)$, we denote by $\gamma_{b}$ the positive function defined by $\gamma_{b}(t)=b\left(\max \left\{t, \frac{1}{t}\right\}\right)$ for all $t>0$.

Lemma 3.1 Let $b$ be a slowly varying function on $[1,+\infty)$.

(i) Let $\epsilon>0$. Then $t^{\epsilon} b(t) \rightarrow+\infty$ as $t \rightarrow+\infty$, and $t^{-\epsilon} b(t) \rightarrow 0$ as $t \rightarrow+\infty$.

(ii) Let $\alpha>0$ and let $b_{1}$ be the positive function on $[1,+\infty)$ defined by $b_{1}(t)=b\left(t^{\alpha}\right)$ for all $t \geq 1$. Then $b_{1}$ is a slowly varying function on $[1,+\infty)$.

Proof. The proof is straightforward.

Let $\alpha \in(0,1]$. Let $\mathcal{K}_{\alpha}$ be the class of all positive and Lebesgue-measurable functions $b$ defined on $[1,+\infty)$ such that, for each $\epsilon>0, \exp \left(\epsilon \ell_{1}^{\alpha}(t)\right) b(t)$ is equivalent to a non-decreasing function on $[1,+\infty)$ and $\exp \left(-\epsilon \ell_{1}^{\alpha}(t)\right) b(t)$ is equivalent to a non-increasing function on $[1,+\infty)$. This class was introduced in [19], where some properties were presented.

Let $p, q \in(0,+\infty]$ and let $b$ be a slowly varying function on $[1,+\infty)$. The Lorentz-Karamata (LK) space $L_{p, q ; b}(R)$ is defined to be the set of all functions $f \in \mathcal{M}_{0}(R, \mu)$ such that

$$
\|f\|_{p, q ; b ; R}:=\left\|t^{\frac{1}{p}-\frac{1}{q}} \gamma_{b}(t) f^{*}(t)\right\|_{q ;(0,+\infty)}
$$

is finite. Here $\|\cdot\|_{q ;(0,+\infty)}$ stands for the usual $L_{q}$ (quasi-)norm over the interval $(0,+\infty)$. When $0<p<+\infty$, the Lorentz-Karamata space $L_{p, q ; b}(R)$ contains the characteristic function of every measurable subset of $R$ with finite measure and hence, by linearity, every $\mu$-simple function; when $p=+\infty$, the Lorentz-Karamata space $L_{p, q ; b}(R)$ is different from the trivial space if, and only if, $\left\|t^{\frac{1}{p}-\frac{1}{q}} \gamma_{b}(t)\right\|_{q ;(0,1)}<+\infty$. We refer to [19] for more details.

When $1<p \leq+\infty$ and $1 \leq q \leq+\infty, L_{p, q ; b}(R)$ can be endowed with a norm $\|\cdot\|_{(p, q ; b) ; R}$ equivalent to $\|.\|_{p, q ; b ; R}$, where $\|f\|_{(p, q ; b) ; R}$ is defined similarly to (3.1) save $f^{*}$ is replaced by $f^{* *}$, see [19, Lemma 3.5].

If we consider $m \in \mathbb{N}, \boldsymbol{\alpha}=\left(\alpha_{1}, \ldots, \alpha_{m}\right) \in \mathbb{R}^{m}$ and $b=\boldsymbol{\ell}^{\boldsymbol{\alpha}}$, then $L_{p, q ; b}(R)$ is precisely the generalised Lorentz-Zygmund (GLZ) $L_{p, q ; \boldsymbol{\alpha}}(R)$, introduced in [7], endowed with the (quasi-)norm $\|f\|_{p, q ; \boldsymbol{\alpha} ; R}$. We remark that in [7], the generalised Lorentz-Zygmund (GLZ) space $L_{p, q ; \boldsymbol{\alpha}}(R)$ and the quasi-norm $\|.\|_{p, q ; \boldsymbol{\alpha} ; R}$ defined above are denoted by $L_{p, q ; \alpha_{1}, \ldots, \alpha_{m}}(R)$ and $\|.\|_{p, q ; \alpha_{1}, \ldots, \alpha_{m} ; R}$, respectively. We use the notation in [7] only when we are considering particular cases. Let us observe that when we consider $\boldsymbol{\alpha}=(0, \ldots, 0)$, we obtain the Lorentz space $L_{p, q}(R)$ endowed with the (quasi-)norm $\|\cdot\|_{p, q ; R}$, which is just the Lebesgue space $L_{p}(R)$ endowed with the (quasi-)norm $\|\cdot\|_{p ; R}$ when $p=q$; if $p=q, m=1$ and $(R, \mu)=\left(\Omega, \mu_{n}\right)$, we obtain the Zygmund space $L^{p}(\log L)^{\alpha_{1}}(\Omega)$ endowed with the (quasi-)norm $\|\cdot\|_{p ; \alpha_{1} ; \Omega}$.

Lemma 3.2 Let $1<p<+\infty, 1 \leq q<+\infty$ and let $b$ be a slowly varying function on $[1,+\infty)$. Then the space $X=\left(L_{p, q ; b}(R),\|\cdot\|_{(p, q ; b) ; R}\right)$ has absolutely continuous norm.

Proof. The proof is like that of [7, Lemma 3.10], where a similar result was established but for generalised Lorentz-Zygmund spaces. Let $f \in X$. Then Lemma 3.7 of [19] gives

$$
f^{*}(t) \leq f^{* *}(t) \precsim \frac{t^{-\frac{1}{p}}}{\gamma_{b}(t)}\|f\|_{p, q ; b ; R}, \quad t>0 .
$$


This entails $f^{*}(t)<+\infty$ for all $t \in(0,+\infty)$, and together with Lemma 3.1 (i) yields $\lim _{t \rightarrow+\infty} f^{*}(t)=0$. Let $\left\{E_{n}\right\}_{n}$ be an arbitrary decreasing sequence of subsets of $R$ such that $E_{n} \downarrow \emptyset$, that is, $\chi_{E_{n}} \downarrow 0 \mu$-a.e. Set $f_{n}=f \chi_{E_{n}}$ for each $n \in \mathbb{N}$. Then $\lim _{n \rightarrow+\infty} f_{n}=0 \mu$-a.e. and

$$
\left|f_{n}\right| \leq|f| \text { for all } n \in \mathbb{Z}
$$

By [7, Corollary 3.9], $\lim _{n \rightarrow+\infty} f_{n}^{*}=0^{*}=0$. From (3.2) we have $f_{n}^{*} \leq f^{*}$ and thus by [19, Lemma 3.5] and by Lebesgue's dominated convergence theorem,

$$
\left\|f \chi_{E_{n}}\right\|_{X}=\left\|f_{n}\right\|_{X} \approx\left(\int_{0}^{+\infty}\left(t^{\frac{1}{p}-\frac{1}{q}} \gamma_{b}(t) f_{n}^{*}(t)\right)^{q} d t\right)^{\frac{1}{q}} \longrightarrow 0
$$

as $n \rightarrow+\infty$, and the result now follows.

We are going to need a variant of the Marcinkiewicz interpolation theorem for the Lorentz-Karamata spaces defined on $\mathbb{R}^{n}$. We refer to [7, Theorem 3.14 \& Corollary 3.15] for the case of generalised Lorentz-Zygmund spaces; see [10] for an exhaustive treatment of this for generalised Lorentz-Zygmund spaces for the case $m=2$ and with the spaces defined over a finite measure space with non-atomic measure.

Suppose that $1 \leq p_{1}<p_{2} \leq+\infty$ and that $q_{1}, q_{2} \in[1,+\infty], q_{1} \neq q_{2}$. Let $\Sigma$ stand for the interpolation segment $\left[\left(\frac{1}{p_{1}}, \frac{1}{q_{1}}\right),\left(\frac{1}{p_{2}}, \frac{1}{q_{2}}\right)\right]$, by which we mean that $\Sigma$ is the line segment with endpoints $\left(\frac{1}{p_{i}}, \frac{1}{q_{i}}\right), i=1,2$. Put

$$
\ell=\frac{\frac{1}{q_{1}}-\frac{1}{q_{2}}}{\frac{1}{p_{1}}-\frac{1}{p_{2}}}
$$

For each $g \in \mathcal{M}^{+}(0,+\infty)$ and each $t \in(0,+\infty)$ the Calderón operator $S_{\Sigma}$ associated with the interpolation segment $\Sigma$ is given by

$$
S_{\Sigma}(g)(t)=t^{-1 / q_{1}} \int_{0}^{t^{\ell}} \tau^{\frac{1}{p_{1}}-1} g(\tau) d \tau+t^{-1 / q_{2}} \int_{t^{\ell}}^{+\infty} \tau^{\frac{1}{p_{2}}-1} g(\tau) d \tau
$$

Now let $T$ be a quasi-linear operator with values in $\mathcal{M}\left(\mathbb{R}^{n}, \mu_{n}\right)$ and defined for all those $f$ in $\mathcal{M}_{0}\left(\mathbb{R}^{n}, \mu_{n}\right)$ for which

$$
S_{\Sigma}\left(f^{*}\right)(1)<+\infty
$$

(We recall that $T$ is called quasi-linear if there is a positive constant $\kappa \geq 1$ such that for all $f$ and $g$ in the domain of $T$, and all scalars $\lambda,|T(f+g)| \leq \kappa(|T f|+|T g|),|T(\lambda f)|=|\lambda||T f|$.) Then $T$ is said to be of joint weak type $\left(p_{1}, q_{1} ; p_{2}, q_{2}\right)$ if, for each $t \in(0,+\infty),(T(f))^{*}(t) \precsim S_{\Sigma}\left(f^{*}\right)(t)$ for all $f$ satisfying (3.4).

The main interpolation result which we shall need is the following:

Theorem 3.3 Let $1 \leq p_{1}<p_{2} \leq+\infty$ and let $q_{1}, q_{2} \in[1,+\infty]$ such that $q_{1} \neq q_{2}$; let $1 \leq r \leq s \leq+\infty$ and let $b$ be a slowly varying function on $[1,+\infty)$. Suppose that $\theta \in(0,1)$ and let $p, q$ given by

$$
\frac{1}{p}=\frac{1-\theta}{p_{1}}+\frac{\theta}{p_{2}}, \quad \frac{1}{q}=\frac{1-\theta}{q_{1}}+\frac{\theta}{q_{2}} .
$$

Let $T$ be a quasi-linear operator of joint weak type $\left(p_{1}, q_{1} ; p_{2}, q_{2}\right)$ and suppose

$$
\sup _{1<t<+\infty} \frac{b_{\ell}(t)}{b(t)}<+\infty
$$

where $\ell$ is given by (3.3) and $b_{\ell}$ is the slowly varying function on $[1,+\infty)$ defined by $b_{\ell}(t)=b\left(t^{1 /|\ell|}\right)$ for all $t \in[1,+\infty)$. Then $T$ maps $L_{p, r ; b}\left(\mathbb{R}^{n}\right)$ boundedly to $L_{q, s ; b}\left(\mathbb{R}^{n}\right)$. 
Proof. It follows that of Theorem 3.4 in [7] by taking into account that $\gamma_{b}\left(t^{1 / \ell}\right)=\gamma_{b}\left(t^{1 /|\ell|}\right)=\gamma_{b_{\ell}}(t)$ for each $t \in(0,+\infty)$, and the Hardy-type inequalities from [19, Lemma 3.2].

Corollary 3.4 Let $T$ be a quasi-linear operator such that for all $q \in(1,+\infty)$,

$$
T: L_{q}\left(\mathbb{R}^{n}\right) \longrightarrow L_{q}\left(\mathbb{R}^{n}\right)
$$

is bounded. Let $1<p<+\infty, 1 \leq r \leq+\infty$ and let b be a slowly varying function on $[1,+\infty)$. Then

$$
T: L_{p, r ; b}\left(\mathbb{R}^{n}\right) \longrightarrow L_{p, r ; b}\left(\mathbb{R}^{n}\right)
$$

is bounded.

Proof. Choose $p_{1}, p_{2}$ so that $1<p_{1}<p<p_{2}<+\infty$ and define $\theta \in(0,1)$ by $\frac{1}{p}=\frac{1-\theta}{p_{1}}+\frac{\theta}{p_{2}}$. By (3.6), $T$ is of strong types $\left(p_{1}, p_{1}\right)$ and $\left(p_{2}, p_{2}\right)$. Hence, by [2, Theorem IV.4.11], $T$ is of joint weak type $\left(p_{1}, p_{1} ; p_{2}, p_{2}\right)$. Since $\ell=1, b_{\ell}=b$ and the result now follows from Theorem 3.3.

\section{Besov-Lipschitz-Karamata spaces}

We start by introducing the Besov-Lipschitz-Karamata spaces $\Lambda_{p, q}^{\lambda, b}\left(\mathbb{R}^{n}\right)$, with $1 \leq p \leq+\infty, 0<q \leq+\infty$, $\lambda \in(0,1]$ and $b$ a slowly varying function on $[1,+\infty)$. The indices $\lambda$ and $b$ are called the first and the second indices of smoothness, respectively. These spaces are a particular case of the general Besov-Hölder-Lipschitz spaces considered in [17].

Let $1 \leq p \leq+\infty$ and $0<q \leq+\infty$. Let $\lambda \in(0,1]$ and let $b$ be a slowly varying function on $[1,+\infty)$. Suppose that $\left\|t^{1-\lambda-\frac{1}{q}} \frac{1}{b(1 / t)}\right\|_{q ;(0,1)}<+\infty$, if $\lambda=1$. The Besov-Lipschitz-Karamata space $\Lambda_{p, q}^{\lambda, b}\left(\mathbb{R}^{n}\right)$ consists of all those functions $f$ in $Y_{p}\left(\mathbb{R}^{n}\right)$ for which the quasi-norm $\left\|f \mid \Lambda_{p, q}^{\lambda, b}\right\|$ is finite, where

$$
\left\|f \mid \Lambda_{p, q}^{\lambda, b}\right\|=\|f\|_{p}+\left(\int_{0}^{1}\left(\frac{\omega(f, t)_{p}}{t^{\lambda} b(1 / t)}\right)^{q} \frac{d t}{t}\right)^{1 / q}
$$

if $0<q<+\infty$, and

$$
\left\|f \mid \Lambda_{p, \infty}^{\lambda, b}\right\|=\|f\|_{p}+\sup _{0<t<1} \frac{\omega(f, t)_{p}}{t^{\lambda} b(1 / t)} .
$$

Remark 4.1 It is easy to see that if we take in the previous definition the range of $t$ to be $(0, \delta)$, for some $\delta \in(0,1]$, instead of $(0,1)$, we obtain an equivalent quasi-norm. Note also that

$$
\left\|f \mid \Lambda_{p, q}^{\lambda, b}\right\|=\|f\|_{p}+\left\|t^{1-\lambda-\frac{1}{q}} \gamma_{\frac{1}{b}}(t) \widetilde{\omega}(f, t)_{p}\right\|_{q ;(0,1)} \quad \text { for all } \quad f \in \Lambda_{p, q}^{\lambda, b}\left(\mathbb{R}^{n}\right)
$$

When $\lambda=1$, a suitable notation for $\Lambda_{p, q}^{1, b}\left(\mathbb{R}^{n}\right)$ would be $\operatorname{Lip} p_{p, q}^{1, b}\left(\mathbb{R}^{n}\right)$. If $\lambda=1$ and $b \approx 1$, i.e. $b$ is a constant function, then $\Lambda_{\infty, \infty}^{1, b}\left(\mathbb{R}^{n}\right)=\Lambda_{\infty, \infty}^{1,1}\left(\mathbb{R}^{n}\right)$ is the subspace of $C\left(\mathbb{R}^{n}\right)$ of the Lipschitz functions and is denoted by $\operatorname{Lip}\left(\mathbb{R}^{n}\right)$. When $\lambda \in(0,1)$, a suitable notation for $\Lambda_{p, q}^{\lambda, b}\left(\mathbb{R}^{n}\right)$ would be $B_{p, q}^{\lambda, b}\left(\mathbb{R}^{n}\right)$.

Remark 4.2 If either $\lambda>1$ or $\lambda=1$ and $\left\|t^{1-\lambda-\frac{1}{q}} \gamma_{\frac{1}{b}}(t)\right\|_{q ;(0,1)}=+\infty$, we have $\Lambda_{p, q}^{\lambda, b}\left(\mathbb{R}^{n}\right)=\{0\}$, if $1 \leq p<+\infty$, and $\Lambda_{\infty, q}^{\lambda, b}\left(\mathbb{R}^{n}\right)$ consisting only of constant functions on $\mathbb{R}^{n}$. Let $\lambda=1$. When $m \in \mathbb{Z}, \boldsymbol{\alpha} \in \mathbb{R}^{m}$ and $b=\ell^{\boldsymbol{\alpha}},\left\|t^{1-\lambda-\frac{1}{q}} \gamma_{\frac{1}{b}}(t)\right\|_{q ;(0,1)}$ is finite if, and only if, either $\boldsymbol{\alpha} \succ \frac{1}{q}+\mathbf{0}$ and $0<q<+\infty$ or $\boldsymbol{\alpha} \succeq \mathbf{0}$ and $q=+\infty$; see [19, Remark 3.6]. We shall denote the space $\Lambda_{p, q}^{1, b}\left(\mathbb{R}^{n}\right)$ by either $\Lambda_{p, q}^{(1, \boldsymbol{\alpha})}\left(\mathbb{R}^{n}\right)$ or $\operatorname{Lip}(1,-\boldsymbol{\alpha})\left(\mathbb{R}^{n}\right)$. When $m=1, b=\ell_{1}^{\alpha}$, with $\alpha>\frac{1}{q}(\alpha \geq 0$, if $q=+\infty)$, cf. Remark 18 in [14], the spaces $\Lambda_{p, q}^{1, b}\left(\mathbb{R}^{n}\right)$ give rise to the spaces $\operatorname{Lip}(1,-\alpha)\left(\mathbb{R}^{n}\right)$ considered in [14].

We refer to [17], where the general Besov-Hölder-Lipschitz spaces $\Lambda_{p, q}^{\rho}\left(\mathbb{R}^{n}\right)$, with $\rho$ a $q$-admissible function, are considered.

Extentions of Besov and Lipschitz spaces have been studied in great detail by the Russian school, mainly Gol'dman [11], [12], [13] and Kalyabin [15]. 
Theorem 4.3 Let $1 \leq p \leq+\infty$ and $0<q \leq+\infty$. Let $\lambda \in(0,1]$ and let $b$ be a slowly varying function on $[1,+\infty)$. Suppose that $\left\|t^{1-\lambda-\frac{1}{q}} \frac{1}{b(1 / t)}\right\|_{q ;(0,1)}<+\infty$, if $\lambda=1$. Then $\Lambda_{p, q}^{\lambda, b}\left(\mathbb{R}^{n}\right)$, endowed with the quasi-norm $\left\|\cdot \mid \Lambda_{p, q}^{\lambda, b}\right\|$, is a quasi-Banach space.

Proof. The proof of the completeness is quite standard. It is a consequence of the completeness of $Y_{p}\left(\mathbb{R}^{n}\right)$, the fact that $\omega(., t)_{p}$ is a semi-norm and $\omega(., t)_{p} \leq 2\|\cdot\|_{p}$ (for each fixed $t \in(0,+\infty)$ ), and Fatou's Lemma; see [18, proof of Theorem 3.1.4].

The next proposition shows that we could have defined the space $\Lambda_{\infty, \infty}^{\lambda, b}\left(\mathbb{R}^{n}\right)$ as a subspace of the uniformly continuous functions on $\mathbb{R}^{n}$.

Proposition 4.4 Let $\lambda \in(0,1]$ and let $b$ be a slowly varying function on $[1,+\infty)$. Suppose that $\left\|t^{1-\lambda} \frac{1}{b(1 / t)}\right\|_{\infty ;(0,1)}<+\infty$, if $\lambda=1$. Then $\Lambda_{\infty, \infty}^{\lambda, b}\left(\mathbb{R}^{n}\right) \hookrightarrow C\left(\overline{\mathbb{R}^{n}}\right)$.

Pro of. It is straightforward by taking into consideration Lemma 3.1 (i).

A simpler characterisation of $\Lambda_{\infty, \infty}^{\lambda, b}\left(\mathbb{R}^{n}\right)$ is given by the next proposition, which is a particular case of [17, Proposition 3.5].

Proposition 4.5 Let $\lambda \in(0,1]$ and let $b$ be a slowly varying function on $[1,+\infty)$. Suppose that $\left\|t^{1-\lambda} \frac{1}{b(1 / t)}\right\|_{\infty ;(0,1)}<+\infty$, if $\lambda=1$. Let $f \in C\left(\mathbb{R}^{n}\right)$. Then $f \in \Lambda_{\infty, \infty}^{\lambda, b}\left(\mathbb{R}^{n}\right)$ if, and only if,

$$
\sup _{\substack{x, y \in \mathbb{R}^{n} \\ 0<|x-y|<1}} \frac{|f(x)-f(y)|}{|x-y|^{\lambda} \gamma_{b}(|x-y|)}<+\infty .
$$

Moreover, for each $f \in \Lambda_{\infty, \infty}^{\lambda, b}\left(\mathbb{R}^{n}\right)$,

$$
\left\|f \mid \Lambda_{\infty, \infty}^{\lambda, b}\right\| \approx\|f\|_{\infty}+\sup _{\substack{x, y \in \mathbb{R}^{n} \\ 0<|x-y|<1}} \frac{|f(x)-f(y)|}{|x-y|^{\lambda} \gamma_{b}(|x-y|)} .
$$

We refer to [18, Sub-section 3.1.2] for sufficient conditions on the indices $\lambda, q$ and on the slowly varying functions $b$ in order to have embeddings between Besov-Lipschitz-Karamata spaces. Alternatively, the proof of these results follow that of embeddings concerning Lorentz-Karamata spaces by taking into consideration that $\widetilde{\omega}(f, .)_{p}$ is equivalent to a non-increasing function, see [19]. We refer to [14, Proposition 16] for embeddings between the spaces $\operatorname{Lip}_{p, q}^{(1,-\alpha)}\left(\mathbb{R}^{n}\right)$.

\section{Bessel-potential-type embedding theorems}

In this section we deal with embedding results for certain Bessel-potential spaces modelled upon LorentzKaramata spaces in the super-limiting case. Thus when $p$ is in the limiting case, i.e., $p=\frac{n}{\sigma}$, where $\sigma \in(0, n)$, and the slowly varying function satisfies a super-limiting condition (see (5.8)), $H^{\sigma} X$ is embedded in $C\left(\mathbb{R}^{n}\right)$, where $C\left(\mathbb{R}^{n}\right)$ denotes the space of bounded and continuous scalar-valued functions defined on $\mathbb{R}^{n}$; when $p$ is in the super-limiting case, i.e., $p>\frac{n}{\sigma}$, where $\sigma \in(0,+\infty), H^{\sigma} X$ is again embedded in $C\left(\mathbb{R}^{n}\right)$. However in this case more can be said. Embeddings in Besov-Lipschitz-Karamata spaces are possible. These results extend those of Edmunds, Gurka and Opic [7] in the context of logarithmic Bessel potential spaces. Moreover, when $p=\frac{n}{\sigma-1}, \sigma \in(1, n+1)$, we improve the results of Edmunds, Gurka and Opic [7, Theorem 4.11] and present refinements of a result due to Triebel [22, Theorem 14.2 (ii)] (but in the context of Bessel potential spaces rather than the Triebel-Lizorkin spaces) which itself improves the result of Brézis-Wainger (super-limiting case) about the "almost Lipschitz continuity" of elements of $H_{p}^{1+n / p}\left(\mathbb{R}^{n}\right)$, cf. [4, Corollary 5].

Let $\sigma>0, p \in(1,+\infty), q \in[1,+\infty]$, and $b$ a s.v. function on $[1,+\infty)$. The Lorentz-Karamata-Bessel potential space $H^{\sigma} L_{p, q ; b}\left(\mathbb{R}^{n}\right)$ is defined to be

$$
\left\{u: u=g_{\sigma} * f, f \in L_{p, q ; b}\left(\mathbb{R}^{n}\right)\right\}
$$


and is equipped with the (quasi-)norm $\|u\|_{\sigma ; p, q ; b}:=\|f\|_{p, q ; b}$.

For $\sigma=0$, we put $H^{\sigma} L_{p, q ; b}\left(\mathbb{R}^{n}\right)=L_{p, q ; b}\left(\mathbb{R}^{n}\right)$.

When we consider $m \in \mathbb{N}, \boldsymbol{\alpha}=\left(\alpha_{1}, \ldots, \alpha_{m}\right) \in \mathbb{R}^{m}$ and $b=\ell^{\boldsymbol{\alpha}}$, we obtain the logarithmic Bessel potential space $H^{\sigma} L_{p, q ; \boldsymbol{\alpha}}\left(\mathbb{R}^{n}\right)$, endowed with the (quasi-)norm $\|u\|_{\sigma ; p, q ; b}$, considered in [7]. Note that if $\boldsymbol{\alpha}=(0, \ldots, 0)$, $H^{\sigma} L_{p, p ; \boldsymbol{\alpha}}\left(\mathbb{R}^{n}\right)$ is simply the (fractional) Sobolev space $H_{p}^{\sigma}\left(\mathbb{R}^{n}\right)$ of order $\sigma$.

When $k \in \mathbb{N}$ we define, using standard notation,

$$
W^{k} L_{p, q ; b}\left(\mathbb{R}^{n}\right)=\left\{u: D^{\alpha} u \in L_{p, q ; b}\left(\mathbb{R}^{n}\right), \text { if }|\alpha| \leq k\right\},
$$

and equip this space with the (quasi-)norm $\sum_{|\alpha| \leq k}\left\|D^{\alpha} u\right\|_{p, q ; b}$.

First we are concerned with the relation between $H^{\sigma} L_{p, q ; b}\left(\mathbb{R}^{n}\right)$, when $\sigma$ is a natural number $k$, and the space $W^{k} L_{p, q ; b}\left(\mathbb{R}^{n}\right)$. It is well-known that if $1<p<+\infty$, then the Bessel potential space $H^{k} L_{p}\left(\mathbb{R}^{n}\right)$, when $k \in \mathbb{N}$, coincides with the Sobolev space $W_{p}^{k}\left(\mathbb{R}^{n}\right)$. Edmunds, Gurka and Opic [7, Theorem 4.2] also proved that an analogous assertion holds when the role of the Lebesgue spaces is played by the generalised Lorentz-Zygmund spaces $L_{p, q ; \boldsymbol{\alpha}}\left(\mathbb{R}^{n}\right)$, with $\boldsymbol{\alpha} \in \mathbb{R}^{m}$ and $p, q \in(1,+\infty)$. We also have that a similar result holds if we consider Lorentz-Karamata spaces instead of generalised Lorentz-Zygmund spaces. The basic tool needed to establish this is Lemma 5.2 which extends [7, Lemma 4.1] and [20, Chap. V, Lemma 3].

We shall need some density results, which generalise [7, Lemma 3.12].

Lemma 5.1 Let $1<p<+\infty, 1 \leq q<+\infty$ and let $b$ be a slowly varying function on $[1,+\infty)$. Then

(i) $C_{0}^{\infty}\left(\mathbb{R}^{n}\right)$ is dense in $L_{p, q ; b}\left(\mathbb{R}^{n}\right)$;

(ii) $C_{0}^{\infty}\left(\mathbb{R}^{n}\right)$ is dense in $W^{1} L_{p, q ; b}\left(\mathbb{R}^{n}\right)$;

(iii) the Schwartz space $S\left(\mathbb{R}^{n}\right)$ is dense in $H^{\sigma} L_{p, q ; b}\left(\mathbb{R}^{n}\right)$ for all $\sigma \geq 0$.

Pro of. By Theorem 3.1 of [19] and Lemma 3.2, $L_{p, q ; b}\left(\mathbb{R}^{n}\right)$ is a rearrangement-invariant function space with absolutely continuous norm. The proof of (i) and (ii) follows by [7, Remark 3.13], where it is observed that parts (i) and (ii) hold if instead of $L_{p, q ; b}\left(\mathbb{R}^{n}\right)$ we consider any Banach function space $L=L\left(\mathbb{R}^{n}\right)$ which is rearrangement-invariant with respect to Lebesgue measure on $\mathbb{R}^{n}$ and which has absolutely continuous norm. Let us now prove part (iii). We start by proving that, under our conditions, $S\left(\mathbb{R}^{n}\right) \subset L_{p, q ; b}\left(\mathbb{R}^{n}\right)$. Let $f \in S\left(\mathbb{R}^{n}\right)$. Then for each $m \in \mathbb{N}_{0}$, there exists $c_{m} \in(0,+\infty)$ such that

$$
|f(x)| \leq \frac{c_{m}}{\left(1+|x|^{2}\right)^{m / 2}} \quad \text { for all } \quad x \in \mathbb{R}^{n} .
$$

On the other hand,

$$
\begin{aligned}
& \int_{0}^{+\infty}\left(t^{\frac{1}{p}-\frac{1}{q}} \gamma_{b}(t) f^{*}(t)\right)^{q} d t \\
& \quad=\int_{0}^{1}\left(t^{\frac{1}{p}-\frac{1}{q}} \gamma_{b}(t) f^{*}(t)\right)^{q} d t+\int_{1}^{+\infty}\left(t^{\frac{1}{p}-\frac{1}{q}} \gamma_{b}(t) f^{*}(t)\right)^{q} d t
\end{aligned}
$$

From (5.1), with $m=0$, we have $f^{*}(t) \leq c_{0}$ for all $t \in(0,+\infty)$. Then

$$
\int_{0}^{1}\left(t^{\frac{1}{p}-\frac{1}{q}} \gamma_{b}(t) f^{*}(t)\right)^{q} d t \leq c_{0}^{q} \int_{0}^{1} t^{\frac{q}{p}-1} \gamma_{b}^{q}(t) d t<+\infty .
$$

From (5.1), with $m \geq 1$ to be chosen later, we have

$$
f^{*}(t) \leq c_{m} \frac{\omega_{n}^{m / n}}{\left(\omega_{n}^{2 / n}+t^{2 / n}\right)^{m / 2}} \text { for all } t \in[0,+\infty),
$$

where $\omega_{n}$ is the volume of the unit ball in $\mathbb{R}^{n}$. Then

$$
\int_{1}^{+\infty}\left(t^{\frac{1}{p}-\frac{1}{q}} \gamma_{b}(t) f^{*}(t)\right)^{q} d t \precsim \int_{1}^{+\infty} t^{-\frac{q m}{n}+\frac{q}{p}-1} \gamma_{b}^{q}(t) d t .
$$


If we choose $m \in \mathbb{N}$ such that $m>\frac{n}{p}$, then the right-hand side of (5.4) is finite. Now, it follows from (5.2), (5.3) and (5.4) that $f \in L_{p, q ; b}\left(\mathbb{R}^{n}\right)$, which gives $S\left(\mathbb{R}^{n}\right) \subset L_{p, q ; b}\left(\mathbb{R}^{n}\right)$. Next, let us prove that for any $\sigma>0$, $S\left(\mathbb{R}^{n}\right) \subset H^{\sigma} L_{p, q ; b}\left(\mathbb{R}^{n}\right)$. By [20, pp. 135], $\left\{g_{\sigma} * h: h \in S\left(\mathbb{R}^{n}\right)\right\}=S\left(\mathbb{R}^{n}\right)$ for any $\sigma>0$. Let $\sigma>0$ and $f \in S\left(\mathbb{R}^{n}\right)$. Then there exists $h \in S\left(\mathbb{R}^{n}\right)$ such that $f=g_{\sigma} * h$. But since $S\left(\mathbb{R}^{n}\right) \subset L_{p, q ; b}\left(\mathbb{R}^{n}\right)$, it now follows that $h \in L_{p, q ; b}\left(\mathbb{R}^{n}\right)$ and therefore $f \in H^{\sigma} L_{p, q ; b}\left(\mathbb{R}^{n}\right)$, which proves that $S\left(\mathbb{R}^{n}\right) \subset H^{\sigma} L_{p, q ; b}\left(\mathbb{R}^{n}\right)$. The density now follows from part (i).

Lemma 5.2 Let $\sigma \in[1,+\infty), p \in(1,+\infty), q \in(1,+\infty)$ and let $b$ be a slowly varying function on $[1,+\infty)$. Then $f \in H^{\sigma} L_{p, q ; b}\left(\mathbb{R}^{n}\right)$ if, and only if, $f \in H^{\sigma-1} L_{p, q ; b}\left(\mathbb{R}^{n}\right)$ and the distributional derivatives $\frac{\partial f}{\partial x_{j}}$ belong to $H^{\sigma-1} L_{p, q ; b}\left(\mathbb{R}^{n}\right)(j=1, \ldots, n)$.

Moreover, the (quasi-)norms

$$
\|f\|_{\sigma ; p, q ; b} \quad \text { and } \quad\|f\|_{\sigma-1 ; p, q ; b}+\sum_{j=1}^{n}\left\|\frac{\partial f}{\partial x_{j}}\right\|_{\sigma-1 ; p, q ; b}
$$

are equivalent on $H^{\sigma} L_{p, q ; b}\left(\mathbb{R}^{n}\right)$.

Proof. In view of Lemma 5.1 and Corollary 3.4, the proof can be carried out as in the case of fractional Sobolev spaces $H_{p}^{\sigma}\left(\mathbb{R}^{n}\right)$ (see [20, Chap. V, Lemma 3]).

The promised result now follows directly

Theorem 5.3 Let $k \in \mathbb{N}, p \in(1,+\infty), q \in(1,+\infty)$ and let b be a slowly varying function on $[1,+\infty)$. Then

$$
H^{k} L_{p, q ; b}\left(\mathbb{R}^{n}\right)=W^{k} L_{p, q ; b}\left(\mathbb{R}^{n}\right)
$$

and the corresponding (quasi-)norms are equivalent.

Pr o of. Similar to that of [20, Chap. V, Theorem 3].

We shall be concerned now with the embedding results to which we referred above. We start by recalling some results needed in the sequel.

The next Lemma, which is a combination of two results due to Edmunds, Gurka and Opic, cf. [6, Lemma 3.5] and [7, (4.23) in Corollary 4.6], provides us estimates for the non-increasing rearrangement of the Bessel Kernel.

Lemma 5.4 Let $\sigma \in(0,+\infty)$. Then there exists a constant $B \in(0,+\infty)$ such that

$$
g_{\sigma}^{*}(t) \precsim t^{\sigma / n-1} \exp \left(-B t^{1 / n}\right), \text { if } \sigma \in(0, n) \text { and } t \in(0,+\infty) \text {, }
$$

and such that

$$
g_{\sigma}^{*}(t) \precsim \begin{cases}\exp \left(-B t^{1 / n}\right) \ell_{1}(t), & \text { if } \sigma=n \text { and } t \in(0,1), \\ \exp \left(-B t^{1 / n}\right), & \text { if } \sigma>n \text { and } t \in(0,1), \\ \exp \left(-B t^{1 / n}\right), & \text { if } \sigma \geq n \text { and } t \in[1,+\infty) .\end{cases}
$$

We shall make use of the following result due to Edmunds, Gurka and Opic.

Lemma 5.5 [7, Lemma 4.5] Let $Y=Y\left(\mathbb{R}^{n}\right)$ be a Banach function space, with absolutely continuous norm, which is rearrangement-invariant (with respect to Lebesgue measure on $\mathbb{R}^{n}$ ). Suppose that $\sigma>0$ and $\left\|g_{\sigma}\right\|_{Y^{\prime}}<+\infty$, where $g_{\sigma}$ is the Bessel kernel. Then

$$
H^{\sigma} Y \hookrightarrow C\left(\mathbb{R}^{n}\right)
$$

The next result extends [7, Corollary 4.6 \& Remark 4.7], concerning the logarithmic Bessel potential space. 
Proposition 5.6 Let $q \in[1,+\infty)$ and let $b$ be a slowly varying function on $[1,+\infty)$. Suppose that either

or

$$
\sigma \in(0, n), \quad p=\frac{n}{\sigma} \quad \text { and } \quad\left\|t^{-\frac{1}{q^{\prime}}} \gamma_{\frac{1}{b}}(t)\right\|_{q^{\prime} ;(0,1)}<+\infty
$$

$$
\sigma \in(0,+\infty), \quad \max \left\{1, \frac{n}{\sigma}\right\}<p<+\infty
$$

Then

$$
H^{\sigma} L_{p, q ; b}\left(\mathbb{R}^{n}\right) \hookrightarrow C\left(\mathbb{R}^{n}\right)
$$

Pro of. We follow the proof of [7, Corollary 4.6]. Let $Y\left(\mathbb{R}^{n}\right)=L_{p, q ; b}\left(\mathbb{R}^{n}\right)$. In view of Lemma 5.5, we need only to show that $\left\|g_{\sigma}\right\|_{Y^{\prime}}<+\infty$.

Suppose first that (5.8) holds. Then, by [19, Theorem 3.1]

$$
Y^{\prime}=L_{\frac{n}{n-\sigma}, q^{\prime} ; \frac{1}{b}}\left(\mathbb{R}^{n}\right) \text {. }
$$

Thus by (5.10), (5.8) and (5.5),

$$
\left\|g_{\sigma}\right\|_{Y^{\prime}}=\left\|t^{1-\frac{\sigma}{n}-\frac{1}{q^{\prime}}} \gamma_{\frac{1}{b}}(t) g_{\sigma}^{*}(t)\right\|_{q^{\prime} ;(0,+\infty)} \precsim I_{1}+I_{2}
$$

where

$$
I_{1}=\left\|t^{-\frac{1}{q^{\prime}}} \gamma_{\frac{1}{b}}(t) \exp \left(-B t^{1 / n}\right)\right\|_{q^{\prime} ;(0,1)}, \quad I_{2}=\left\|t^{-\frac{1}{q^{\prime}}} \gamma_{\frac{1}{b}}(t) \exp \left(-B t^{1 / n}\right)\right\|_{q^{\prime} ;(1,+\infty)} .
$$

Plainly $I_{2}<+\infty$, due to the exponential factor and because $b$ is a slowly varying function on $[1,+\infty)$. Since $\left\|t^{-\frac{1}{q^{\prime}}} \gamma_{\frac{1}{b}}(t)\right\|_{q^{\prime} ;(0,1)}<+\infty$, it also follows that $I_{1} \leq\left\|t^{-\frac{1}{q^{\prime}}} \gamma_{\frac{1}{b}}(t)\right\|_{q^{\prime} ;(0,1)}<+\infty$.

Now suppose that (5.9) holds. Then, by [19, Theorem 3.1], $Y^{\prime}=L_{p^{\prime}, q^{\prime} ; \frac{1}{b}}\left(\mathbb{R}^{n}\right)$. If $\sigma \in(0, n)$, we obtain from (5.5) that

$$
\left\|g_{\sigma}\right\|_{Y^{\prime}}=\left\|t^{\frac{1}{p^{\prime}}-\frac{1}{q^{\prime}}} \gamma_{\frac{1}{b}}(t) g_{\sigma}^{*}(t)\right\|_{q^{\prime} ;(0,+\infty)} \precsim J_{1}+J_{2}
$$

where

$$
\begin{aligned}
& J_{1}=\left\|t^{\frac{1}{p^{\prime}}+\frac{\sigma}{n}-1-\frac{1}{q^{\prime}}} \gamma_{\frac{1}{b}}(t) \exp \left(-B t^{1 / n}\right)\right\|_{q^{\prime} ;(0,1)}, \\
& J_{2}=\left\|t^{\frac{1}{p^{\prime}}+\frac{\sigma}{n}-1-\frac{1}{q^{\prime}}} \gamma_{\frac{1}{b}}(t) \exp \left(-B t^{1 / n}\right)\right\|_{q^{\prime} ;(1,+\infty)} .
\end{aligned}
$$

Once more, due to the exponential factor and because $b$ is a slowly varying function on $[1,+\infty)$ it is clear that $J_{2}<+\infty$. Since our assumptions imply that $\frac{1}{p^{\prime}}+\frac{\sigma}{n}-1>0$, we have by [19, Lemma 3.1 (v)] that $J_{1} \leq\left\|t^{\frac{1}{p^{\prime}}+\frac{\sigma}{n}-1-\frac{1}{q^{\prime}}} \gamma_{\frac{1}{b}}(t)\right\|_{q^{\prime} ;(0,1)}<+\infty$. Hence $\left\|g_{\sigma}\right\|_{Y^{\prime}}<+\infty$. To deal with the case in which $\sigma \in[n,+\infty)$, we use the estimates (5.6). Therefore, we obtain

$$
\left\|g_{\sigma}\right\|_{Y^{\prime}}=\left\|t^{\frac{1}{p^{\prime}}-\frac{1}{q^{\prime}}} \gamma_{\frac{1}{b}}(t) g_{\sigma}^{*}(t)\right\|_{q^{\prime} ;(0,+\infty)} \precsim K_{1}+K_{2},
$$

where

$$
K_{1}= \begin{cases}\left\|t^{\frac{1}{p^{\prime}}-\frac{1}{q^{\prime}}} \gamma_{\frac{1}{b}}(t) \ell_{1}(t) \exp \left(-B t^{1 / n}\right)\right\|_{q^{\prime} ;(0,1)}, & \text { if } \quad \sigma=n, \\ \left\|t^{\frac{1}{p^{\prime}}-\frac{1}{q^{\prime}}} \gamma_{\frac{1}{b}}(t) \exp \left(-B t^{1 / n}\right)\right\|_{q^{\prime} ;(0,1)}, & \text { if } \sigma>n .\end{cases}
$$

and

$$
K_{2}=\left\|t^{\frac{1}{p^{\prime}}-\frac{1}{q^{\prime}}} \gamma_{\frac{1}{b}}(t) \exp \left(-B t^{1 / n}\right)\right\|_{q^{\prime} ;(1,+\infty)}
$$


Again, due to the exponential factor and because $b$ is a slowly varying function on $[1,+\infty)$, it is easy to verify that $K_{2}<+\infty$. As for $K_{1}$, let us observe the following. Let $b_{1}$ be the slowly varying function defined by $b_{1}(t)=\frac{\ell_{1}(t)}{b(t)}$ for each $t \geq 1$. Then $\gamma_{b_{1}}(t)=\gamma_{\frac{1}{b}}(t) \ell_{1}(t)$ for each $t \in(0,+\infty)$ and

$$
K_{1} \leq \begin{cases}\left\|t^{\frac{1}{p^{\prime}}-\frac{1}{q^{\prime}}} \gamma_{b_{1}}(t)\right\|_{q^{\prime} ;(0,1)}, & \text { if } \quad \sigma=n, \\ \left\|t^{\frac{1}{p^{\prime}}-\frac{1}{q^{\prime}}} \gamma_{\frac{1}{b}}(t)\right\|_{q^{\prime} ;(0,1)}, & \text { if } \quad \sigma>n .\end{cases}
$$

Therefore, since $\frac{1}{p^{\prime}}>0$, we have by [19, Lemma 3.1(v)] that $K_{1}<+\infty$, and the result now follows.

Remark 5.7 Let $q \in[1,+\infty), m \in \mathbb{N}, \boldsymbol{\alpha} \in \mathbb{R}^{m}$ and $b=\ell^{\boldsymbol{\alpha}}$.

(i) Then $\left\|t^{-\frac{1}{q^{\prime}}} \gamma_{\frac{1}{b}}(t)\right\|_{q^{\prime} ;(0,1)}<+\infty$ if, and only if, either $1<q<\infty$ and $\boldsymbol{\alpha} \succ \frac{1}{q^{\prime}}+\mathbf{0}$ or $q=1$ and $\boldsymbol{\alpha} \succeq \mathbf{0}$.

(ii) In the counterpart of (5.8) of [7, Corollary $4.6 \&$ Remark 4.7], only the case $\boldsymbol{\alpha} \succ \frac{1}{q^{\prime}}+\mathbf{0}$, with $q \in[1,+\infty)$ is considered. However, the result of Proposition 5.6 still holds with $\boldsymbol{\alpha}=\mathbf{0}$ and $q=1$ in (5.8).

Remark 5.8 Let $m \in \mathbb{N}, q \in[1,+\infty), \alpha_{1} \in \mathbb{R}$ and $0<\alpha<1$. Let $\phi \in \mathcal{K}_{\alpha}$ and let $b$ be the slowly varying function on $[1,+\infty)$ defined by

$$
b_{1}(t)=\ell_{m}^{-\frac{\alpha-1}{q^{\prime}}}(t) \prod_{i=1}^{m-1} \ell_{i}^{\frac{1}{q^{\prime}}}(t) \exp \left(\alpha_{1} \ell_{m}^{\alpha}(t)\right) \phi\left(\ell_{m-1}(t)\right) \quad \text { for each } \quad t \geq 1 .
$$

(i) If $\phi$ is a constant function, i.e. $\phi(t) \approx 1$ for each $t \geq 1$, then

$$
\left\|t^{-\frac{1}{q^{\prime}}} \gamma_{\frac{1}{b}}(t)\right\|_{q^{\prime} ;(0,1)}<+\infty
$$

if, and only if, either $1<q<\infty$ and $\alpha_{1}>0$ or $q=1$ and $\alpha_{1} \geq 0$.

(ii) If $\alpha_{1}>0$, then $\left\|t^{-\frac{1}{q^{\prime}}} \gamma_{\frac{1}{b}}(t)\right\|_{q^{\prime} ;(0,1)}<+\infty$. If $\alpha_{1}<0$, then $\left\|t^{-\frac{1}{q^{\prime}}} \gamma_{\frac{1}{b}}(t)\right\|_{q^{\prime} ;(0,1)}=+\infty$.

The next result is an extension of [7, Theorem 4.8] and a refinement of [16, Theorem 5.7.7 (i)].

Theorem 5.9 [19, Theorem 5.1] Let $\sigma \in(0, n), 1<p<\frac{n}{\sigma}, q \in[1,+\infty]$ and let $b$ be a slowly varying function on $[1,+\infty)$. Then

$$
H^{\sigma} L_{p, q ; b}\left(\mathbb{R}^{n}\right) \hookrightarrow L_{r, q ; b}\left(\mathbb{R}^{n}\right),
$$

where $\frac{1}{r}=\frac{1}{p}-\frac{\sigma}{n}$.

Although a direct proof of Theorem 5.9 is given in [19], note that the result is also a consequence of Theorem 3.3, because the operator $T$ defined by $T f=u=g_{\sigma} * f$ is of joint weak type $\left(p_{1}, q_{1} ; p_{2}, q_{2}\right)$, with $1 / q_{1}=$ $(n-\sigma) / n, p_{1}=1, q_{2}=+\infty$ and $1 / p_{2}=\sigma / n$; see the proof of Theorem 4.8 in [7] for more details.

Theorem 5.9 enables us to prove the next result, which is a refinement of [16, Theorem 5.7.8(i)] and an extension of [7, Theorem 4.9].

Theorem 5.10 Suppose that $1<q<+\infty, \sigma \in[1, n+1), \max \left\{1, \frac{n}{\sigma}\right\}<p<\frac{n}{\sigma-1}$. Let b be a slowly varying function on $[1,+\infty)$. Then for any $u \in H^{\sigma} L_{p, q ; b}\left(\mathbb{R}^{n}\right)$ and all $x, y \in \mathbb{R}^{n}$,

$$
|u(x)-u(y)| \precsim\|u\|_{\sigma ; p, q ; b}|x-y|^{\sigma-\frac{n}{p}} \gamma_{\frac{1}{b}}\left(|x-y|^{n}\right) .
$$

Moreover,

$$
H^{\sigma} L_{p, q ; b}\left(\mathbb{R}^{n}\right) \hookrightarrow \Lambda_{\infty, \infty}^{\lambda, b_{1}}\left(\mathbb{R}^{n}\right)
$$

where $\lambda:=\sigma-\frac{n}{p} \in(0,1)$ and $b_{1}$ is the slowly varying function on $[1,+\infty)$ defined by $b_{1}(t)=\frac{1}{b\left(t^{n}\right)}$ for all $t \in[1,+\infty)$. 
Proof. In view of Lemma 5.1 and Proposition 5.6, it is enough to prove (5.12) for any $u \in S\left(\mathbb{R}^{n}\right)$. Let $x, y \in \mathbb{R}^{n}$ and suppose $x \neq y$, otherwise the result is trivial. Let $\rho \in(0,+\infty)$ such that $0<|x-y|=\rho$. Then there is a cube $Q_{\rho}$ of edge length $\rho$ such that $x, y \in \bar{Q}_{\rho}$. Given any $u \in S\left(\mathbb{R}^{n}\right)$ and $z \in Q_{\rho}$, we have

$$
u(x)-u(z)=\int_{0}^{1} \sum_{i=1}^{n} \frac{\partial u}{\partial x_{i}}(x+t(z-x))\left(z_{i}-x_{i}\right) d t .
$$

This implies

$$
u(x)-\rho^{-n} \int_{Q_{\rho}} u(z) d z=\rho^{-n} \int_{Q_{\rho}} \int_{0}^{1} \sum_{i=1}^{n} \frac{\partial u}{\partial x_{i}}(x+t(z-x))\left(z_{i}-x_{i}\right) d t d z
$$

Hence,

$$
\begin{aligned}
\left|u(x)-\rho^{-n} \int_{Q_{\rho}} u(z) d z\right| & =\rho^{-n}\left|\int_{Q_{\rho}} \int_{0}^{1} \sum_{i=1}^{n} \frac{\partial u}{\partial x_{i}}(x+t(z-x))\left(z_{i}-x_{i}\right) d t d z\right| \\
& \leq \sqrt{n} \int_{0}^{1} \rho^{1-n} t^{-n}\left(\sum_{i=1}^{n} \int_{Q_{t \rho}^{x}}\left|\frac{\partial u}{\partial x_{i}}(s)\right| d s\right) d t
\end{aligned}
$$

where for each $t \in(0,1)$ the symbol $Q_{t \rho}^{x}$ denotes a sub-cube of $Q_{\rho}$ with faces parallel to those of $Q_{\rho}$, with side length $t \rho$, and which is given by $Q_{t \rho}^{x}=\left\{s \in Q_{\rho}: s=x+t(z-x), z \in Q_{\rho}\right\}$. Since $u \in S\left(\mathbb{R}^{n}\right) \subset$ $H^{\sigma} L_{p, q ; b}\left(\mathbb{R}^{n}\right)$, Lemma 5.2 shows that

$$
\frac{\partial u}{\partial x_{i}} \in H^{\sigma-1} L_{p, q ; b}\left(\mathbb{R}^{n}\right), \quad i=1, \ldots, n .
$$

If $\sigma>1$ then, by Theorem 5.9, with $\sigma-1$ instead of $\sigma, H^{\sigma-1} L_{p, q ; b}\left(\mathbb{R}^{n}\right) \hookrightarrow L_{r, q ; b}\left(\mathbb{R}^{n}\right)$, where $\frac{1}{r}=\frac{1}{p}-\frac{\sigma-1}{n}$. If $\sigma=1$, then $r=p$ and $H^{\sigma-1} L_{p, q ; b}\left(\mathbb{R}^{n}\right) \hookrightarrow L_{r, q ; b}\left(\mathbb{R}^{n}\right)$ holds trivially. Thus Hölder's inequality, cf. [2, Corollary II.4.5], and [19, Theorem 3.1] yield

$$
\sum_{i=1}^{n} \int_{Q_{t \rho}^{x}}\left|\frac{\partial u}{\partial x_{i}}(s)\right| d s \leq \sum_{i=1}^{n}\left\|\frac{\partial u}{\partial x_{i}}\right\|\left\|_{r, q ; b}\right\| \chi_{Q_{t \rho}^{x}} \|_{r^{\prime}, q^{\prime} ; \frac{1}{b}} .
$$

Furthermore,

$$
\sum_{i=1}^{n}\left\|\frac{\partial u}{\partial x_{i}}\right\|_{r, q ; b} \precsim \sum_{i=1}^{n}\left\|\frac{\partial u}{\partial x_{i}}\right\|_{\sigma-1 ; p, q ; b} \precsim\|u\|_{\sigma ; p, q ; b},
$$

and because $\frac{1}{r^{\prime}}>0,[19$, Lemma $3.1(\mathrm{v})]$ gives

$$
\left\|\chi_{Q_{t \rho}^{x}}\right\|_{r^{\prime}, q^{\prime} ; \frac{1}{b}}=\left\|\tau^{\frac{1}{r^{\prime}}-\frac{1}{q^{\prime}}} \gamma_{\frac{1}{b}}(\tau) \chi_{\left(0,(t \rho)^{n}\right)}(\tau)\right\|_{q^{\prime} ;(0,+\infty)} \approx(t \rho)^{n / r^{\prime}} \gamma_{\frac{1}{b}}\left((t \rho)^{n}\right)
$$

for each $t \in(0,1)$. Hence

$$
\int_{0}^{1} \rho^{1-n} t^{-n}\left(\sum_{i=1}^{n} \int_{Q_{t \rho}^{x}}\left|\frac{\partial u}{\partial x_{i}}(s)\right| d s\right) d t \precsim\|u\|_{\sigma ; p, q ; b} I(\rho),
$$

where $I(\rho)=\frac{1}{n} \int_{0}^{\rho^{n}} \tau^{\frac{\sigma}{n}-\frac{1}{p}-1} \gamma_{\frac{1}{b}}(\tau) d \tau$. Since $\frac{\sigma}{n}-\frac{1}{p}>0$, [19, Lemma 3.1(v)] yields $I(\rho) \approx \rho^{\sigma-\frac{n}{p}} \gamma_{\frac{1}{b}}\left(\rho^{n}\right)$, which together with (5.14) and (5.18) ensure

$$
\left|u(x)-\rho^{-n} \int_{Q_{\rho}} u(z) d z\right| \precsim\|u\|_{\sigma ; p, q ; b} \rho^{\sigma-\frac{n}{p}} \gamma_{\frac{1}{b}}\left(\rho^{n}\right),
$$


and so $|u(x)-u(y)| \precsim\|u\|_{\sigma ; p, q ; b}|x-y|^{\sigma-\frac{n}{p}} \gamma_{\frac{1}{b}}\left(|x-y|^{n}\right)$, for all $x, y \in \mathbb{R}^{n}$ and the proof of (5.12) is complete.

Let us now prove (5.13). Let $u \in H^{\sigma} L_{p, q ; b}\left(\mathbb{R}^{n}\right)$. From (5.12), we have

$$
|u(x)-u(y)| \precsim\|u\|_{\sigma ; p, q ; b}|x-y|^{\sigma-\frac{n}{p}} \gamma_{\frac{1}{b_{1}}}(|x-y|)
$$

for all $x, y \in \mathbb{R}^{n}$ such that $0<|x-y|<1$, where $b_{1}$ is the slowly varying function on $[1,+\infty)$ defined by $b_{1}(t)=\frac{1}{b\left(t^{n}\right)}$ for all $t \in[1,+\infty)$. On the other hand, cf. Proposition 5.6, $u \in C\left(\mathbb{R}^{n}\right)$ and $\|u\|_{\infty} \precsim\|u\|_{\sigma ; p, q ; b}$. Then the assertion now follows from Proposition 4.5.

Pro of. The previous proof was carried out as that of [7, Theorem 4.9], by taking into consideration Lemma 5.1, Proposition 5.6, Lemma 5.2, Theorem 5.1 of [19], [2, Corollary II.4.5], Theorem 3.1 of [19], Lemma 3.1 (v) of [19] and Proposition 4.5.

Remark 5.11 Let us assume the conditions of Theorem 5.10 hold. Let $m \in \mathbb{N}, \boldsymbol{\alpha} \in \mathbb{R}^{m}$ and let $b=\ell^{\boldsymbol{\alpha}}$. Then $b_{1} \approx \ell^{-\alpha}$ and in this case the previous theorem coincides with [7, Theorem 4.9] and with the first part of [7, Theorem 4.16].

The next result is a refinement of [16, Theorem 5.7.8 (iii)] and extends [7, Theorem 4.13].

Theorem 5.12 Let $1<q<+\infty$ and let b be a slowly varying function on $[1,+\infty)$. Suppose that either

or

$$
\sigma \in(1, n+1), \quad p=\frac{n}{\sigma-1} \quad \text { and } \quad\left\|t^{-\frac{1}{q^{\prime}}} \gamma_{\frac{1}{b}}(t)\right\|_{q^{\prime} ;(0,1)}<+\infty
$$

$$
\sigma \in(1,+\infty), \quad \max \left\{1, \frac{n}{\sigma-1}\right\}<p<+\infty
$$

Then for any $u \in H^{\sigma} L_{p, q ; b}\left(\mathbb{R}^{n}\right)$ and all $x, y \in \mathbb{R}^{n}$,

$$
|u(x)-u(y)| \precsim\|u\|_{\sigma ; p, q ; b}|x-y| .
$$

Moreover,

$$
H^{\sigma} L_{p, q ; b}\left(\mathbb{R}^{n}\right) \hookrightarrow \Lambda_{\infty, \infty}^{1,1}\left(\mathbb{R}^{n}\right)=\operatorname{Lip}\left(\mathbb{R}^{n}\right) .
$$

Proof. In view of Lemma 5.1 and Proposition 5.6, it is enough to prove (5.21) for any $u \in S\left(\mathbb{R}^{n}\right)$. Let $x, y \in \mathbb{R}^{n}$ and suppose $x \neq y$, otherwise the result is trivial. Let $\rho \in(0,+\infty)$ such that $0<|x-y|=\rho$. Then we have (5.14). In view of (5.15) and Proposition 5.6 (with $\sigma-1$ instead of $\sigma$ ), it follows that $\frac{\partial u}{\partial x_{i}} \in L_{\infty}\left(\mathbb{R}^{n}\right)$. Also, with the same notation as in the proof of Theorem 5.10, Hölder's inequality yields

$$
\sum_{i=1}^{n} \int_{Q_{t \rho}^{x}}\left|\frac{\partial u}{\partial x_{i}}(s)\right| d s \leq \sum_{i=1}^{n}\left\|\frac{\partial u}{\partial x_{i}}\right\|_{\infty}\left\|\chi_{Q_{t \rho}^{x}}\right\|_{1} .
$$

Furthermore,

$$
\sum_{i=1}^{n}\left\|\frac{\partial u}{\partial x_{i}}\right\|_{\infty} \precsim \sum_{i=1}^{n}\left\|\frac{\partial u}{\partial x_{i}}\right\|_{\sigma-1 ; p, q ; b} \precsim\|u\|_{\sigma ; p, q ; b},
$$

and

$$
\left\|\chi_{Q_{t \rho}^{x}}\right\|_{1}=\int_{0}^{(t \rho)^{n}} d \tau=(t \rho)^{n}
$$

for each $t \in(0,1)$. From (5.14), (5.23), (5.24) and (5.25) we have

$$
\left|u(x)-\rho^{-n} \int_{Q_{\rho}} u(z) d z\right| \precsim\|u\|_{\sigma ; p, q ; b} \int_{0}^{1} \rho^{1-n} t^{-n}(t \rho)^{n} d t=\rho\|u\|_{\sigma ; p, q ; b} .
$$

Thus $|u(x)-u(y)| \precsim\|u\|_{\sigma ; p, q ; b}|x-y|$ for all $x, y \in \mathbb{R}^{n}$ and the proof of (5.21) is complete. 
Let us now prove (5.22). Let $u \in H^{\sigma} L_{p, q ; b}\left(\mathbb{R}^{n}\right)$. From (5.21), we have

$$
|u(x)-u(y)| \precsim\|u\|_{\sigma ; p, q ; b}|x-y|
$$

for all $x, y \in \mathbb{R}^{n}$ such that $0<|x-y|<1$. On the other hand, $u \in C\left(\mathbb{R}^{n}\right)$ and $\|u\|_{\infty} \precsim\|u\|_{\sigma ; p, q ; b}(\mathrm{cf}$. Proposition 5.6). Then the result now follows from Theorem 4.5.

We refer to Remark 5.8 for other examples of slowly varying functions for which Theorem 5.12 holds. These examples have not been considered before in the literature, as far as we are aware.

In order to consider further embedding results, which are going to improve and extend those of Edmunds, Gurka and Opic concerning logarithmic Bessel potential spaces, we shall need the next two propositions. Proposition 5.13 gives an important estimate due to Triebel, cf. [22, Proposition 12.16(i)].

Proposition 5.13 Let $\epsilon \in(0,1)$. Then there is a number $c>0$ such that

$$
\widetilde{\omega}(f, t)_{\infty} \leq c|\nabla f|^{* *}\left(t^{2 n-1}\right)+3 \sup _{0<\tau \leq t^{2}} \tau^{-1 / 2} \omega(f, \tau)_{\infty}
$$

for all $t \in(0, \epsilon)$ and all $f \in C^{1}\left(\overline{\mathbb{R}^{n}}\right)$, where $\nabla f=\left(\frac{\partial f}{\partial x_{1}}, \ldots, \frac{\partial f}{\partial x_{n}}\right)$ and $|\nabla f|$ denotes the Euclidean norm of $\nabla f$.

The next result extends [22, Proposition 12.16(ii)], restricted here however to the case $q \in[1,+\infty]$.

Proposition 5.14 Let $q \in[1,+\infty]$ and let $b$ be a slowly varying function on $[1,+\infty)$ such that $\left\|t^{-\frac{1}{q}} \gamma_{b}(t)\right\|_{q ;(0,1)}<+\infty$. Then

$$
\begin{aligned}
\left\|t^{-\frac{1}{q}} \gamma_{b_{1}}(t) \widetilde{\omega}(f, t)_{\infty}\right\|_{q ;(0,1)} & \precsim\left\|t^{-\frac{1}{q}} \gamma_{b}(t)|\nabla f|^{* *}(t)\right\|_{q ;(0,1)} \\
& \approx\left\|t^{-\frac{1}{q}} \gamma_{b}(t)|\nabla f|^{*}(t)\right\|_{q ;(0,1)}
\end{aligned}
$$

for all $f \in C^{1}\left(\overline{\mathbb{R}^{n}}\right)$, where $b_{1}$ is the slowly varying function on $[1,+\infty)$ defined by $b_{1}(t)=b\left(t^{2 n-1}\right)$ for all $t \in[1,+\infty)$.

Proof. We use the main ideas in the proof of [22, Proposition 12.16(ii)], where the case $\gamma_{b_{1}}(t) \approx \gamma_{b}(t) \approx$ $|\ln t|^{-u}$ for each $t \in(0, \epsilon)$, with $0<\epsilon<1, u=\frac{1}{q}+v, v>0$ and the range of $t$ in (5.27) is equal to $(0, \epsilon)$, is considered.

Suppose $1 \leq q<+\infty$ and let $\epsilon \in(0,1)$ to be chosen later. Let $f \in C^{1}\left(\overline{\mathbb{R}^{n}}\right)$. By (5.26) from Proposition 5.13, we have

$$
\begin{aligned}
& \left\|t^{-\frac{1}{q}} \gamma_{b_{1}}(t) \widetilde{\omega}(f, t)_{\infty}\right\|_{q ;(0, \epsilon)} \\
& \quad \precsim\left\|t^{-\frac{1}{q}} \gamma_{b_{1}}(t)\right\|_{q ;(0,1)} \sup _{0<t<\epsilon} t^{\frac{1}{2}} \widetilde{\omega}(f, t)_{\infty}+\left\|t^{-\frac{1}{q}} \gamma_{b_{1}}(t)|\nabla f|^{* *}\left(t^{2 n-1}\right)\right\|_{q ;(0, \epsilon)} .
\end{aligned}
$$

Now

$$
\left\|t^{-\frac{1}{q}} \gamma_{b_{1}}(t)\right\|_{q ;(0,1)} \approx\left\|t^{-\frac{1}{q}} \gamma_{b}(t)\right\|_{q ;(0,1)}<+\infty
$$

and, since $\widetilde{\omega}(f, .)_{\infty}$ is equivalent to a non-increasing function on $(0,+\infty)$, we have

$$
\sup _{0<t<\epsilon} t^{\frac{1}{2}} \widetilde{\omega}(f, t)_{\infty} \precsim\left(\int_{0}^{\epsilon}\left(t^{\frac{1}{2}-\frac{1}{q}} \widetilde{\omega}(f, t)_{\infty}\right)^{q} d t\right)^{\frac{1}{q}} .
$$

So from (5.28), (5.29), (5.30) and by the change of variables $\tau=t^{2 n-1}$, we obtain

$$
\begin{aligned}
& \left\|t^{-\frac{1}{q}} \gamma_{b_{1}}(t) \widetilde{\omega}(f, t)_{\infty}\right\|_{q ;(0, \epsilon)} \\
& \quad \precsim\left\|t^{\frac{1}{2}-\frac{1}{q}} \widetilde{\omega}(f, t)_{\infty}\right\|_{q ;(0, \epsilon)}+\left\|t^{-\frac{1}{q}} \gamma_{b_{1}}(t)|\nabla f|^{* *}\left(t^{2 n-1}\right)\right\|_{q ;(0, \epsilon)} \\
& \quad \leq c_{1}\left\|t^{\frac{1}{2}-\frac{1}{q}} \widetilde{\omega}(f, t)_{\infty}\right\|_{q ;(0, \epsilon)}+c_{2}\left\|\tau^{-\frac{1}{q}} \gamma_{b}(\tau)|\nabla f|^{* *}(\tau)\right\|_{q ;(0,1)},
\end{aligned}
$$


where $c_{1}, c_{2}$ are positive constants. Now, since $b_{1}$ is a slowly varying function on $[1,+\infty)$, there exists $\epsilon_{0} \in(0,1)$ such that

$$
t^{\frac{1}{2}} \leq \frac{1}{2 c_{1}} \gamma_{b_{1}}(t) \text { for each } t \in\left(0, \epsilon_{0}\right)
$$

This is the counterpart of (12.102) in [22]. Now under the additional condition $\epsilon:=\epsilon_{0}$, we have from (5.31) and (5.32) that

Therefore

$$
\begin{aligned}
& \left\|t^{-\frac{1}{q}} \gamma_{b_{1}}(t) \widetilde{\omega}(f, t)_{\infty}\right\|_{q ;(0, \epsilon)} \\
& \quad \leq(1 / 2)\left\|t^{-\frac{1}{q}} \gamma_{b_{1}}(t) \widetilde{\omega}(f, t)_{\infty}\right\|_{q ;(0, \epsilon)}+c_{2}\left\|\tau^{-\frac{1}{q}} \gamma_{b}(\tau)|\nabla f|^{* *}(\tau)\right\|_{q ;(0,1)} .
\end{aligned}
$$

$$
\left\|t^{-\frac{1}{q}} \gamma_{b_{1}}(t) \widetilde{\omega}(f, t)_{\infty}\right\|_{q ;(0, \epsilon)} \precsim\left\|\tau^{-\frac{1}{q}} \gamma_{b}(\tau)|\nabla f|^{* *}(\tau)\right\|_{q ;(0,1)} .
$$

Since $\widetilde{\omega}(f, .)_{\infty}$ is equivalent to a non-increasing function and $\epsilon=\epsilon_{0}<1$, it follows by the change of variables $\tau=\epsilon t$ that

$$
\begin{aligned}
\left\|t^{-\frac{1}{q}} \gamma_{b_{1}}(t) \widetilde{\omega}(f, t)_{\infty}\right\|_{q ;(0,1)} & \precsim\left\|t^{-\frac{1}{q}} \gamma_{b_{1}}(t) \widetilde{\omega}(f, \epsilon t)_{\infty}\right\|_{q ;(0,1)} \\
& \approx\left\|\tau^{-\frac{1}{q}} \gamma_{b_{1}}(\tau) \widetilde{\omega}(f, \tau)_{\infty}\right\|_{q ;(0, \epsilon)} .
\end{aligned}
$$

From (5.33) and (5.34) we then obtain

$$
\left\|t^{-\frac{1}{q}} \gamma_{b_{1}}(t) \widetilde{\omega}(f, t)_{\infty}\right\|_{q ;(0,1)} \precsim\left\|\tau^{-\frac{1}{q}} \gamma_{b}(\tau)|\nabla f|^{* *}(\tau)\right\|_{q ;(0,1)} .
$$

On the other hand, from the Hardy-type inequality (3.6) in [19, Lemma 3.2] with $\nu=-1<0$, we have

$$
\left\|t^{-\frac{1}{q}} \gamma_{b}(t)|\nabla f|^{* *}(t)\right\|_{q ;(0,1)} \precsim\left\|t^{-\frac{1}{q}} \gamma_{b}(t)|\nabla f|^{*}(t)\right\|_{q ;(0,1)} .
$$

By the simple inequality $|\nabla f|^{*}(t) \leq|\nabla f|^{* *}(t)$ for each $t>0$, we have

$$
\left\|t^{-\frac{1}{q}} \gamma_{b}(t)|\nabla f|^{*}(t)\right\|_{q ;(0,1)} \leq\left\|t^{-\frac{1}{q}} \gamma_{b}(t)|\nabla f|^{* *}(t)\right\|_{q ;(0,1)} .
$$

Now (5.27) is a consequence of (5.35), (5.36) and (5.37), when $1 \leq q<+\infty$. The proof with $q=+\infty$ is analogous.

We shall need the following lemma.

Lemma 5.15 [19, Lemma 5.2] Let $\sigma \in(0, n), p, q \in[1,+\infty]$ and let $b_{1}, b_{2}$ be two slowly varying functions on $[1,+\infty)$. Suppose that

and either

$$
\left\|t^{-\frac{1}{q}} b_{2}(1 / t)\right\|_{q ;(0,1)}<+\infty
$$

$$
1 \leq p \leq q \leq+\infty, \quad \sup _{0<x<1} \frac{b_{2}(1 / x)}{b_{1}(1 / x)}<+\infty
$$

and there is a positive constant c such that

$$
\left\|t^{-\frac{1}{q}} b_{2}(1 / t)\right\|_{q ;(0, x)}\left\|\left(t^{\frac{1}{p^{\prime}}} b_{1}(1 / t)\right)^{-1}\right\|_{p^{\prime} ;(x, 1)} \leq c \quad \text { for all } x \in(0,1),
$$

or

$$
1 \leq q<p \leq+\infty, \quad\left\|x^{-\frac{1}{r}} \frac{b_{2}(1 / x)}{b_{1}(1 / x)}\right\|_{r ;(0,1)}<+\infty
$$

and

$$
\int_{0}^{1}\left[\left\|t^{-\frac{1}{q}} b_{2}(1 / t)\right\|_{q ;(0, x)}\left\|\left(t^{\frac{1}{p^{\prime}}} b_{1}(1 / t)\right)^{-1}\right\|_{p^{\prime} ;(x, 1)}^{p^{\prime} / q^{\prime}}\right]^{r}\left(x^{\frac{1}{p^{\prime}}} b_{1}(1 / x)\right)^{-p^{\prime}} d x
$$

is finite, where $\frac{1}{r}=\frac{1}{q}-\frac{1}{p}$. Then

$$
\left\|u^{*}\right\|_{\infty, q ; b_{2} ;(0,1)} \precsim\|u\|_{\sigma ; \frac{n}{\sigma}, p ; b_{1}} \text { holds for all } u \in H^{\sigma} L_{\frac{n}{\sigma}, p ; b_{1}}\left(\mathbb{R}^{n}\right) .
$$


As promised, we are now in a position to present the last embedding results. These results improve and extend [7, Theorem 4.11] and that of Brézis-Wainger about "the almost Lipschitz continuity" of the elements of $H_{p}^{1+n / p}\left(\mathbb{R}^{n}\right)$, i.e., $H_{p}^{1+n / p}\left(\mathbb{R}^{n}\right) \hookrightarrow \operatorname{Lip}_{\infty, \infty}^{\left(1,-\frac{1}{p^{\prime}}\right)}\left(\mathbb{R}^{n}\right)$, cf. [4, Corollary 5]. We were motivated by [22, Theorem 14.2 (ii)], which, with $1<p<+\infty$ and $0<q \leq+\infty$, leads to $F_{p, q}^{1+n / p}\left(\mathbb{R}^{n}\right) \hookrightarrow \operatorname{Lip}_{\infty, p}^{(1,-1)}\left(\mathbb{R}^{n}\right)$. By $F_{p, q}^{1+n / p}\left(\mathbb{R}^{n}\right)$ we mean the Triebel-Lizorkin spaces. Note that Triebel's result improves and extends the above result of Brézis-Wainger about "the almost Lipschitz continuity", because $F_{p, 2}^{1+n / p}\left(\mathbb{R}^{n}\right)$, when $1<p<+\infty$, coincides with $H_{p}^{1+n / p}\left(\mathbb{R}^{n}\right)$, cf. [21, Theorem 2.5.6], and $\operatorname{Lip}_{\infty, p}^{(1,-1)}\left(\mathbb{R}^{n}\right) \hookrightarrow \operatorname{Lip}_{\infty, \infty}^{\left(1,-\frac{1}{p^{\prime}}\right)}\left(\mathbb{R}^{n}\right)$, cf. [14, Proposition 16]. We refer to [22, Chapter II] and to [14] for more results and more details about embeddings of "B" and " $F$ " spaces into spaces of Lipschitz type.

Theorem 5.16 Let $p \in(1,+\infty), q \in[1,+\infty]$ and $\sigma \in(1, n+1)$. Let $b_{1}, b_{2}$ be two slowly varying functions on $[1,+\infty)$ such that

$$
\left\|t^{-\frac{1}{p^{\prime}}} \gamma_{\frac{1}{b_{1}}}(t)\right\|_{p^{\prime} ;(0,1)}=+\infty \quad \text { and }\left\|t^{-\frac{1}{q}} b_{2}(1 / t)\right\|_{q ;(0,1)}<+\infty
$$

and either conditions (5.39), (5.40) or conditions (5.41), (5.42) are verified. Then

$$
H^{\sigma} L_{\frac{n}{\sigma-1}, p ; b_{1}}\left(\mathbb{R}^{n}\right) \hookrightarrow \Lambda_{\infty, q}^{1, b_{3}}\left(\mathbb{R}^{n}\right)=\operatorname{Lip}_{\infty, q}^{1, b_{3}}\left(\mathbb{R}^{n}\right),
$$

where $b_{3}$ is the slowly varying function defined by $b_{3}(t)=\frac{1}{b_{2}\left(t^{2 n-1}\right)}$ for each $t \in[1,+\infty)$.

Proof. Let $u \in S\left(\mathbb{R}^{n}\right) \subset H^{\sigma} L_{\frac{n}{\sigma-1}}, p ; b_{1}\left(\mathbb{R}^{n}\right)$. Then Lemma 5.2 shows that $\frac{\partial u}{\partial x_{i}} \in H^{\sigma-1} L_{\frac{n}{\sigma-1}, p ; b_{1}}\left(\mathbb{R}^{n}\right)$, for $i=1, \ldots, n$. Now by Lemma 5.15, with $\sigma-1$ instead of $\sigma$, and by observing that $v^{*}=|v|^{*}$ for any $v \in \mathcal{M}_{0}\left(\mathbb{R}^{n}, \mu_{n}\right)$, we have

$$
\left\|\left|\frac{\partial u}{\partial x_{i}}\right|^{*}\right\|_{\infty, q ; b_{2} ;(0,1)} \precsim\left\|\frac{\partial u}{\partial x_{i}}\right\|_{\sigma-1 ; \frac{n}{\sigma-1}, p ; b_{1}} \quad \text { for } \quad i=1, \ldots, n .
$$

Hence, by Lemma 5.2,

$$
\sum_{i=1}^{n}\left\|\left|\frac{\partial u}{\partial x_{i}}\right|^{*}\right\|_{\infty, q ; b_{2} ;(0,1)} \precsim \sum_{i=1}^{n}\left\|\frac{\partial u}{\partial x_{i}}\right\|_{\sigma-1 ; \frac{n}{\sigma-1}, p ; b_{1}} \precsim\|u\|_{\sigma ; \frac{n}{\sigma-1}, p ; b_{1}} .
$$

This together with Proposition 5.14 and the Hardy-type inequality (3.6) in [19, Lemma 3.2] with $\nu=-1<0$ give

$$
\begin{aligned}
\left\|t^{-\frac{1}{q}} \gamma_{\frac{1}{b_{3}}}(t) \widetilde{\omega}(u, t)_{\infty}\right\|_{q ;(0,1)} & \precsim\left\|t^{-\frac{1}{q}} \gamma_{b_{2}}(t)|\nabla u|^{* *}(t)\right\|_{q ;(0,1)} \\
& \precsim \sum_{i=1}^{n}\left\|t^{-\frac{1}{q}} \gamma_{b_{2}}(t)\left|\frac{\partial u}{\partial x_{i}}\right|^{* *}(t)\right\|_{q ;(0,1)} \\
& \precsim\|u\|_{\sigma ; \frac{n}{\sigma-1}, p ; b_{1}} .
\end{aligned}
$$

By Proposition 5.6, we also have $\|u\|_{\infty} \precsim\|u\|_{\sigma ; \frac{n}{\sigma-1}, p ; b_{1}}$. This with (5.46) yield

$$
\left\|u \mid \Lambda_{\infty, q}^{1, b_{3}}\right\| \precsim\|u\|_{\sigma ; \frac{n}{\sigma-1}, p ; b_{1}} \quad \text { for all } \quad u \in S\left(\mathbb{R}^{n}\right) .
$$

Now let $u \in H^{\sigma} L_{\frac{n}{\sigma-1}}, p ; b_{1}\left(\mathbb{R}^{n}\right)$. By Lemma 5.1 there exists $\left\{u_{m}\right\}_{m} \subset S\left(\mathbb{R}^{n}\right)$ such that

$$
\left\|u_{m}-u\right\|_{\sigma ; \frac{n}{\sigma-1}, p ; b_{1}} \longrightarrow 0, \quad \text { as } \quad m \rightarrow+\infty .
$$

Hence, $\left\|u_{m}-u_{l}\right\|_{\sigma ; \frac{n}{\sigma-1}, p ; b_{1}} \rightarrow 0$, as $m, l \rightarrow+\infty$, which together with (5.47) yield $\left\|u_{m}-u_{l} \mid \Lambda_{\infty, q}^{1, b_{3}}\right\| \rightarrow 0$ as $m, l \rightarrow+\infty$, i.e., $\left\{u_{m}\right\}_{m}$ is a Cauchy sequence in $\Lambda_{\infty, q}^{1, b_{3}}\left(\mathbb{R}^{n}\right)$. Since $\Lambda_{\infty, q}^{1, b_{3}}\left(\mathbb{R}^{n}\right)$ is a complete space, cf. Theorem 4.3 , there exists $v \in \Lambda_{\infty, q}^{1, b_{3}}\left(\mathbb{R}^{n}\right)$ such that

$$
\left\|u_{m}-v \mid \Lambda_{\infty, q}^{1, b_{3}}\right\| \longrightarrow 0, \text { as } m \rightarrow+\infty .
$$


Now, Proposition 5.6, the trivial embedding $\Lambda_{\infty, q}^{1, b_{3}}\left(\mathbb{R}^{n}\right) \hookrightarrow C\left(\mathbb{R}^{n}\right)$, (5.48) and (5.49) entail $u=v$ and $u_{m} \rightarrow u$, as $m \rightarrow+\infty$ (convergence in $\left.\Lambda_{\infty, q}^{1, b_{3}}\left(\mathbb{R}^{n}\right)\right)$. So, (5.47) gives $\left\|u_{m} \mid \Lambda_{\infty, q}^{1, b_{3}}\right\| \leq\left\|u_{m}\right\|_{\sigma ; \frac{n}{\sigma-1}, p ; b_{1}}$, and by passing to the limit we obtain $\left\|u \mid \Lambda_{\infty, q}^{1, b_{3}}\right\| \leq\|u\|_{\sigma ; \frac{n}{\sigma-1}, p ; b_{1}}$, which yields the assertion.

Remark 5.17 Note that the condition $\left\|t^{-\frac{1}{p^{\prime}}} \gamma_{\frac{1}{b_{1}}}(t)\right\|_{p^{\prime} ;(0,1)}=+\infty$ in (5.44) is not used in the proof of Theorem 5.16, and can therefore be omitted. However, if $\left\|t^{-\frac{1}{p^{\prime}}} \gamma_{\frac{1}{b_{1}}}(t)\right\|_{p^{\prime} ;(0,1)}<+\infty$, we have $H^{\sigma} L_{\frac{n}{\sigma-1}, p ; b_{1}}\left(\mathbb{R}^{n}\right) \hookrightarrow$ $\Lambda_{\infty, \infty}^{1,1}\left(\mathbb{R}^{n}\right)=\operatorname{Lip}\left(\mathbb{R}^{n}\right)$, as results from (5.19) and (5.21) of Theorem 5.12, which is a better result than that given by (5.45). In fact, for any $b_{3}$ and $q \in[1,+\infty]$ under the conditions of Theorem 5.16 , the embedding

$$
\Lambda_{\infty, \infty}^{1,1}\left(\mathbb{R}^{n}\right) \hookrightarrow \Lambda_{\infty, q}^{1, b_{3}}\left(\mathbb{R}^{n}\right)
$$

holds. To see this, note that $\left\|t^{-\frac{1}{q}} \frac{1}{b_{3}(1 / t)}\right\|_{q ;(0,1)} \approx\left\|t^{-\frac{1}{q}} b_{2}(1 / t)\right\|_{q ;(0,1)}<+\infty$, and follow the proof of [19, Theorem 3.2], where embeddings concerning LK spaces were established, by taking into consideration that $\widetilde{\omega}(f, .)_{p}$ is equivalent to a non-increasing function. Alternatively, see [18, Theorem 3.1.7].

Corollary 5.18 Let $p \in(1,+\infty), q \in[1,+\infty]$ and $\sigma \in(1, n+1)$. Let $m \in \mathbb{N}, \boldsymbol{\alpha}=\left(\alpha_{1}, \ldots, \alpha_{m}\right) \in \mathbb{R}^{m}$ and let $k \in\{1, \ldots, m\}$ be such that $\alpha_{k} \neq \frac{1}{p^{\prime}}$ and, if $k \geq 2, \alpha_{i}=\frac{1}{p^{\prime}}, i=1, \ldots, k-1$. Assume $\alpha_{k}<\frac{1}{p^{\prime}}$. Let $\boldsymbol{\beta}=\left(\beta_{1}, \ldots, \beta_{m}\right) \in \mathbb{R}^{m}$ with $\beta_{k} \neq-\frac{1}{q}$ and, if $k \geq 2, \beta_{i}=-\frac{1}{q}, i=1, \ldots, k-1$. Then

$$
H^{\sigma} L_{\frac{n}{\sigma-1}, p ; \boldsymbol{\alpha}}\left(\mathbb{R}^{n}\right) \hookrightarrow \Lambda_{\infty, q}^{(1,-\boldsymbol{\beta})}\left(\mathbb{R}^{n}\right)=\operatorname{Lip}_{\infty, q}^{(1, \boldsymbol{\beta})}\left(\mathbb{R}^{n}\right)
$$

provided one of the following conditions is satisfied:

$$
\begin{aligned}
& 1<p \leq q \leq+\infty, \quad \beta_{k}<-\frac{1}{q}, \quad \boldsymbol{\beta}+\boldsymbol{\delta}_{q ; m, k} \preceq \boldsymbol{\alpha}-\boldsymbol{\delta}_{p^{\prime} ; m, k} ; \\
& 1 \leq q<p<+\infty, \quad \beta_{k}<-\frac{1}{q}, \quad \boldsymbol{\beta}+\frac{1}{q} \prec \boldsymbol{\alpha}-\boldsymbol{\delta}_{1 ; m, k}+\frac{1}{p} .
\end{aligned}
$$

Proof. Let $b_{1}=\ell^{\alpha}$ and $b_{2}=\ell^{\beta}$. Then it follows that both conditions in (5.44) are verified. By [19, Remark 3.2] and [19, Remark 3.4], either conditions (5.39), (5.40) or conditions (5.41), (5.42) are verified. Note that $b_{3}(t) \approx \ell^{-\boldsymbol{\beta}}(t)$ for all $t \in[1,+\infty)$. Hence the result follows from Theorem 5.16.

The situation when $\alpha_{1}=\ldots=\alpha_{m}=\frac{1}{p^{\prime}}$ is also covered by the previous corollary, by using $\widetilde{\boldsymbol{\alpha}}=$ $\left(\widetilde{\alpha}_{1}, \ldots, \widetilde{\alpha}_{m+1}\right) \in \mathbb{R}^{m+1}$ in place of $\boldsymbol{\alpha} \in \mathbb{R}^{m}$, where $\widetilde{\alpha}_{j}=\frac{1}{p^{\prime}}$ for $j=1, \ldots, m$ and $\widetilde{\alpha}_{m+1}=0$, and considering $k=m+1$.

Remark 5.19 (i) When $q=+\infty, k=m$ and $\boldsymbol{\beta}=\boldsymbol{\alpha}-\delta_{p^{\prime} ; m, m}$, the previous corollary gives [7, Therorem 4.11]. In particular, we obtain with $p=n /(\sigma-1), k=m=1, \alpha=0$ and $\beta=-1 / p^{\prime}$, the result of Brézis-Wainger mentioned just before Theorem 5.16.

(ii) When $1<p=q=n /(\sigma-1)<+\infty, k=m=1, \alpha=0$ and $\beta=-1$, we recover the Triebel's result, but in the context of fractional Sobolev spaces; see comments before Theorem 5.16.

(iii) Let $1<p<+\infty, 1 \leq q \leq+\infty, \sigma \in(1, n+1), \boldsymbol{\alpha}, \boldsymbol{\beta} \in \mathbb{R}^{m}, k \in\{1, \ldots, m\}$ as in Corollary 5.18. Suppose additionally that one of the conditions (5.52)-(5.53) is satisfied. Then

$$
H^{\sigma} L_{\frac{n}{\sigma-1}, p ; \boldsymbol{\alpha}}\left(\mathbb{R}^{n}\right) \hookrightarrow \Lambda_{\infty, p}^{(1,-\boldsymbol{\nu})}\left(\mathbb{R}^{n}\right)=\operatorname{Lip}_{\infty, p}^{(1, \boldsymbol{\nu})}\left(\mathbb{R}^{n}\right) \hookrightarrow \Lambda_{\infty, q}^{(1,-\boldsymbol{\beta})}\left(\mathbb{R}^{n}\right)=\operatorname{Lip}_{\infty, q}^{(1, \boldsymbol{\beta})}\left(\mathbb{R}^{n}\right),
$$

where $\boldsymbol{\nu}=\boldsymbol{\alpha}-\boldsymbol{\delta}_{1 ; m, k}$. The first embedding follows from the previous corollary with $q=p$. Since $\boldsymbol{\delta}_{1 ; m, k}=$ $\boldsymbol{\delta}_{p ; m, k}+\boldsymbol{\delta}_{p^{\prime} ; m, k}$ and $\boldsymbol{\nu}=\boldsymbol{\alpha}-\boldsymbol{\delta}_{1 ; m, k}$, the second embedding follows from [18, Theorem 3.1.7] and [18, Remark 3.1.8], if $q \leq p$, and from [18, Theorem 3.1.10] and [18, Remark 3.1.11], if $q>p$. Alternatively, see [19, Remark 5.1] for considerations similar to those regarding the second embedding.

The next corollary presents embeddings of Bessel-Karamata spaces with slowly varying functions different from the ones of the previous corollary, i.e. of logarithmic type. This result has not been considered before in the literature, as far as we are aware. 
Corollary 5.20 Let $p \in(1,+\infty), q \in[1,+\infty]$ and $\sigma \in(1, n+1), m \in \mathbb{N}, \alpha_{1}, \beta_{1} \in \mathbb{R} \backslash\{0\}$ and $\alpha \in(0,1)$. Assume $\alpha_{1}<0$. Let $\phi_{1}, \phi_{2} \in \mathcal{K}_{\alpha}$ and let $b_{1}, b_{2}$ be two slowly varying functions on $[1,+\infty)$ defined by

$$
b_{1}(t)=\ell_{m}^{-\frac{\alpha-1}{p^{\prime}}}(t) \prod_{i=1}^{m-1} \ell_{i}^{\frac{1}{p^{\prime}}}(t) \exp \left(\alpha_{1} \ell_{m}^{\alpha}(t)\right) \phi_{1}\left(\ell_{m-1}(t)\right) \quad \text { for each } \quad t \geq 1 \text {, }
$$

and

$$
b_{2}(t)=\ell_{m}^{\frac{\alpha-1}{q}}(t) \prod_{i=1}^{m-1} \ell_{i}^{-\frac{1}{q}}(t) \exp \left(\beta_{1} \ell_{m}^{\alpha}(t)\right) \phi_{2}\left(\ell_{m-1}(t)\right) \quad \text { for each } t \geq 1,
$$

respectively. Then (5.45) holds, provided one of the following conditions is satisfied:

$$
\begin{aligned}
& 1<p<+\infty, \quad 1 \leq q \leq+\infty, \quad \beta_{1}<\alpha_{1} ; \\
& 1<p \leq q \leq+\infty, \quad \beta_{1}=\alpha_{1}, \quad \phi_{2} \precsim \phi_{1} .
\end{aligned}
$$

Pr o of. By Remark 5.8 and [19, Remarks 3.3, 3.5] the conditions of the previous theorem are satisfied. The result now follows.

Acknowledgements It is a pleasure to thank Prof. D. E. Edmunds for his helpful suggestions during the preparation of this paper. The author is also grateful to Calouste Gulbenkian Foundation and to Mathematics Department of the University of Coimbra for the financial support.

\section{References}

[1] R. A. Adams, Sobolev Spaces (Academic Press, New York, 1975).

[2] C. Bennett and R. Sharpley, Interpolation of Operators, Pure and Applied Mathematics Vol. 129 (Academic Press, New York, 1988).

[3] N. H. Bingham, C. M. Goldie, and J. L. Teugels, Regular Variation (Cambridge University Press, Cambridge, 1987).

[4] H. Brézis and S. Wainger, A note on limiting cases of Sobolev embeddings, Comm. Partial Diff. Equations 5, 773-789 (1980).

[5] R. A. DeVore and G. G. Lorentz, Constructive Approximation, Grundlehren der Mathematischen Wissenschaften - A Series of Comprehensive Studies in Mathematics Vol. 303 (Springer-Verlag, Berlin-Heidelberg, 1993).

[6] D. E. Edmunds, P. Gurka, and B. Opic, Double exponential integrability, Bessel potentials and embedding theorems, Studia Math. 115, 151-181 (1995).

[7] D. E. Edmunds, P. Gurka, and B. Opic, On embeddings of logarithmic Bessel potential spaces, J. Funct. Anal. 146, No. 1, 116-150 (1997).

[8] D. E. Edmunds, P. Gurka, and B. Opic, Optimality of embeddings of logarithmic Bessel potential spaces, Quart. J. Math. 51, 185-209 (2000).

[9] D. E. Edmunds, R. Kerman, and L. Pick, Optimal Sobolev imbeddings involving rearrangement-invariant quasinorms, J. Funct. Anal. 170, 307-355 (2000).

[10] W. D. Evans, B. Opic, and L. Pick, Interpolation of operators on scales of generalised Lorentz-Zygmund spaces, Math. Nachr. 182, 127-181 (1996).

[11] M. L. Gol'dman, Imbedding of generalized Hölder classes, Mat. Zametki 12, 325-336 (1972).

[12] M. L. Gol'dman, A description of the trace space for functions of a generalized Hölder class, Dokl. Akad. Nauk SSSR 231, No. 3, 525-528 (1976). English Translation: Soviet Math. Dokl. 17, No. 6, 1577-1581 (1976).

[13] M. L. Gol'dman, On imbedding of Nikol'skiı̌-Besov spaces with moduli of continuity of general form into Lorentz spaces, Dokl. Akad. Nauk SSSR 277, No. 1, 20-24 (1984). English translation: Soviet Math. Dokl. 30, No. 1, 11-16 (1984).

[14] D. D. Haroske, On more general Lipschitz spaces, Z. Anal. Anwendungen 19, No. 3, 781-800 (2000).

[15] G. A. Kaljabin, Imbedding theorems for generalized Besov and Liouville spaces, Dokl. Akad. Nauk SSSR 232, No. 6, 1245-1248 (1977). English Translation: Soviet Math. Dokl. 18, No. 1, 1577-1581 (1977); Soviet Math. Dokl. 18, No. 4 (1977).

[16] A. Kufner, O. John, and S. Fučík, Function Spaces (Noordhoff International Publishing, Leyden. Academia, Publishing House of the Czechoslovak Academy of Sciences, Prague, 1977).

[17] J. S. Neves, Extrapolation results on general Besov-Hölder-Lipschitz spaces, Math. Nachr. 230, 117-141 (2001).

[18] J. S. Neves, Fractional Sobolev-type spaces and embeddings, Ph.D. thesis, University of Sussex at Brighton (United Kingdom) (2001). 
[19] J. S. Neves, Lorentz-Karamata spaces, Bessel and Riesz potentials and embeddings, Dissertationes Math. (Rozprawy Mat.) 405 (2002).

[20] E. M. Stein, Singular Integrals and Differentiability Properties of Functions (Princeton University Press, Princeton, New Jersey, 1970).

[21] H. Triebel, Theory of Function Spaces, Monographs in Mathematics Vol. 78 (Birkhäuser Verlag, Basel, 1983).

[22] H. Triebel, The structure of functions, Monographs in Mathematics Vol. 97 (Birkhäuser Verlag, Basel, 2001).

[23] A. Zygmund, Trigonometric Series, Vol. I (Cambridge University Press, Cambridge, 1957). 